\title{
REGULARIZED NUMERICAL METHODS FOR THE LOGARITHMIC SCHRÖDINGER EQUATION
}

\author{
WEIZHU BAO, RÉMI CARLES, CHUNMEI SU, AND QINGLIN TANG
}

\begin{abstract}
We present and analyze two numerical methods for the logarithmic Schrödinger equation (LogSE) consisting of a regularized splitting method and a regularized conservative Crank-Nicolson finite difference method (CNFD). In order to avoid numerical blow-up and/or to suppress round-off error due to the logarithmic nonlinearity in the LogSE, a regularized logarithmic Schrödinger equation (RLogSE) with a small regularized parameter $0<\varepsilon \ll 1$ is adopted to approximate the LogSE with linear convergence rate $O(\varepsilon)$. Then we use the LieTrotter splitting integrator to solve the RLogSE and establish its error bound $O\left(\tau^{1 / 2} \ln \left(\varepsilon^{-1}\right)\right)$ with $\tau>0$ the time step, which implies an error bound at $O\left(\varepsilon+\tau^{1 / 2} \ln \left(\varepsilon^{-1}\right)\right)$ for the LogSE by the Lie-Trotter splitting method. In addition, the CNFD is also applied to discretize the RLogSE, which conserves the mass and energy in the discretized level. Numerical results are reported to confirm our error bounds and to demonstrate rich and complicated dynamics of the LogSE.
\end{abstract}

\section{INTRODUCTION}

We consider the logarithmic Schrödinger equation (LogSE) which was originally introduced as a model of nonlinear wave mechanics (cf. 13])

$$
\left\{\begin{array}{l}
i \partial_{t} u(\mathbf{x}, t)+\Delta u(\mathbf{x}, t)=\lambda u(\mathbf{x}, t) \ln \left(|u(\mathbf{x}, t)|^{2}\right), \quad \mathbf{x} \in \Omega, \quad t>0, \\
u(\mathbf{x}, 0)=u_{0}(\mathbf{x}), \quad \mathbf{x} \in \Omega
\end{array}\right.
$$

where $t$ is time, $\mathbf{x}=\left(x_{1}, \ldots, x_{d}\right)^{T} \in \mathbb{R}^{d}(d=1,2,3)$ is the spatial coordinate, $u:=u(\mathbf{x}, t) \in$ $\mathbb{C}$ is the dimensionless wave function, $\lambda \in \mathbb{R} \backslash\{0\}$ is a dimensionless real constant of the nonlinear interaction strength, and $\Omega=\mathbb{R}^{d}$ or $\Omega \subset \mathbb{R}^{d}$ is a bounded domain with homogeneous Dirichlet boundary condition or periodic boundary condition posted on the boundary. The LogSE now arises from different applications, such as quantum mechanics [48], quantum optics [13 15, 31, 38], nuclear physics [33, 35], Bohmian mechanics [41], effective quantum gravity [50], theory of superfluidity and Bose-Einstein condensation [5].

We emphasize that the nonlinearity $z \mapsto z \ln |z|^{2}$ is not locally Lipschitz continuous due to the singularity of the logarithm at the origin, and therefore even the well-posedness of the Cauchy problem for (1.1) is not completely obvious. We refer to $17,21,29$ for a study of the Cauchy problem by compactness methods in a suitable functional framework (which depends on the sign of $\lambda$ based on a priori estimate), and to [32] for an alternative proof relying on the strong convergence of suitable approximate solutions when $\lambda<0$.

This work was partially supported by the Ministry of Education of Singapore grant R-146-000-223-112 (MOE2015-T2-2-146) (W. Bao), and by the Fundamental Research Funds for the Central Universities (Q. Tang). 
Similar to the more usual cubic nonlinear Schrödinger equation, the LogSE conserves the mass, momentum and energy 20], which are defined, respectively, as follows:

$$
\begin{aligned}
M(u(\cdot, t)) & :=\|u(\cdot, t)\|_{L^{2}(\Omega)}^{2} \equiv\left\|u_{0}\right\|_{L^{2}(\Omega)}^{2}=M\left(u_{0}\right), \\
P(u(\cdot, t)) & :=\operatorname{Im} \int_{\Omega} \bar{u}(\mathbf{x}, t) \nabla u(\mathbf{x}, t) d \mathbf{x} \equiv \operatorname{Im} \int_{\Omega} \overline{u_{0}}(\mathbf{x}) \nabla u_{0}(\mathbf{x}) d \mathbf{x}=P\left(u_{0}\right), \quad t \geq 0, \\
E(u(\cdot, t)) & :=\|\nabla u(t)\|_{L^{2}(\Omega)}^{2}+\lambda \int_{\Omega}|u(\mathbf{x}, t)|^{2} \ln \left(|u(\mathbf{x}, t)|^{2}\right) d \mathbf{x} \\
& \equiv\left\|\nabla u_{0}\right\|_{L^{2}(\Omega)}^{2}+\lambda \int_{\Omega}\left|u_{0}(\mathbf{x})\right|^{2} \ln \left(\left|u_{0}(\mathbf{x})\right|^{2}\right) d \mathbf{x}=E\left(u_{0}\right) .
\end{aligned}
$$

At this stage, the only difference with the cubic nonlinear Schrödinger equation is the expression of the energy. We emphasize that the second term in the energy, referred to as interaction energy, has no definite sign, since

$$
\int_{|u|>1}|u(\mathbf{x}, t)|^{2} \ln \left(|u(\mathbf{x}, t)|^{2}\right) d \mathbf{x} \geq 0, \quad \text { while } \quad \int_{|u|<1}|u(\mathbf{x}, t)|^{2} \ln \left(|u(\mathbf{x}, t)|^{2}\right) d \mathbf{x} \leq 0 .
$$

Therefore, it is not obvious to guess which sign of $\lambda$ leads to which type of dynamics. In the case $\lambda<0$, no solution is dispersive, as proven in [19. This is reminiscent of the focusing nonlinear Schrödinger equation, where, however, small solutions are always dispersive. In addition, unlike what happens in the case of the nonlinear Schrödinger equation, every solution is global in time: there is no such thing as finite time blow up for LogSE. In the case $\lambda<0$, stationary solutions are available, called Gaussons (as noticed in [14; see below), which turn out to be orbitally stable, but not stable in the usual sense of Lyapunov 19,22 (see also [4] for another proof, and [44,46] for other particular solutions). In the case $\lambda>0$, every solution is (global and) dispersive with a non-standard rate (a logarithmic perturbation of the Schrödinger rate), and after a time-dependent rescaling related to this dispersion, the large time behavior of the renormalized solution exhibits a universal Gaussian profile - a phenomenon which is rather unique in the context of Hamiltonian dispersive PDEs, see [17.

Note that unlike the more standard nonlinear Schrödinger equation, a large set of explicit solutions is associated to (1.1) in the case $\Omega=\mathbb{R}^{d}$. This important feature was noticed already from the introduction of this model [13]: if $u_{0}$ is Gaussian, $u(\cdot, t)$ is Gaussian for all time, and solving (1.1) amounts to solving ordinary differential equations. For the convenience of the reader, we briefly recall the formulas given in [7]. In the one-dimensional case, if

$$
u_{0}(x)=b_{0} e^{-\frac{a_{0}}{2} x^{2}+i v x}, \quad x \in \mathbb{R},
$$

with $a_{0}, b_{0} \in \mathbb{C}$ satisfying $\alpha_{0}:=\operatorname{Re} a_{0}>0$ and $v \in \mathbb{R}$ being constants, then the solution of (1.1) is given by [4,7, 17]

$$
u(x, t)=\frac{b_{0}}{\sqrt{r(t)}} e^{i\left(v x-v^{2} t\right)+Y(x-2 v t, t)}, \quad x \in \mathbb{R}, \quad t \geq 0,
$$

where

$$
Y(x, t)=-i \phi(t)-\alpha_{0} \frac{x^{2}}{2 r(t)^{2}}+i \frac{\dot{r}(t)}{r(t)} \frac{x^{2}}{4}, \quad x \in \mathbb{R}, \quad t \geq 0
$$


with $\phi:=\phi(t)$ and $r:=r(t)>0$ being the solutions of the ODEs

$$
\begin{aligned}
& \dot{\phi}=\frac{\alpha_{0}}{r^{2}}+\lambda \ln \left|b_{0}\right|^{2}-\lambda \ln r, \quad \phi(0)=0, \\
& \ddot{r}=\frac{4 \alpha_{0}^{2}}{r^{3}}+\frac{4 \lambda \alpha_{0}}{r}, \quad r(0)=1, \quad \dot{r}(0)=-2 \operatorname{Im} a_{0} .
\end{aligned}
$$

In the multi-dimensional case, one can actually tensorize such one-dimensional solutions due to the property $\ln |a b|=\ln |a|+\ln |b|$. In the case $\lambda<0$, if $a_{0}=-\lambda>0$, then $r(t) \equiv 1$, which generates a moving Gausson when $v \neq 0$ and a static Gausson when $v=0$ of the LogSE; and if $0<a_{0} \neq-\lambda$, the function $r$ is (time) periodic (in agreement with the absence of dispersive effects), which generates a breather of the LogSE [7]. In the case $\lambda>0$, the large time behavior of $r$ does not depend on its initial data, $r(t) \sim 2 t \sqrt{\lambda \alpha_{0} \ln t}$ as $t \rightarrow \infty$ (see [17]). The general dynamics is rather well understood in the case $\lambda>0$ (see 17]), but, aside from the explicit Gaussian solutions, the dynamical properties in the case $\lambda<0$ constitute a vast open problem: is the dynamics comparable to the one, say, of the cubic nonlinear Schrödinger equation, or is it drastically different? The numerical simulations presented in this paper tend to suggest that the dynamics associated to (1.1) is quite rich, and reveals phenomena absent (or at least unknown) in the case of the cubic nonlinear Schrödinger equation.

There is a long list of references on numerical approaches for solving the nonlinear Schrödinger (NLS) equation with power-like nonlinearity

$$
i \partial_{t} u(\mathbf{x}, t)+\Delta u(\mathbf{x}, t)=\lambda|u(\mathbf{x}, t)|^{2 \sigma} u(\mathbf{x}, t), \quad \mathbf{x} \in \mathbb{R}^{d}, \quad t>0
$$

with $\sigma \in \mathbb{N}$, or with nonlocal Hartree-type nonlinearity

$$
i \partial_{t} u(\mathbf{x}, t)+\Delta u(\mathbf{x}, t)=\lambda\left(|\mathbf{x}|^{-\gamma} *|u(\mathbf{x}, t)|^{2}\right) u(\mathbf{x}, t), \quad \mathbf{x} \in \mathbb{R}^{d}, \quad t>0
$$

with $\gamma>0$, such as finite difference method [1,3, 23, finite element method [2,37], relaxation method [11], and time-splitting pseudospectral method [3,8,9,40,43, 45, 47], etc. For the error analysis of the time-splitting or split-step method for the NLS equation, we refer to [12, 24, 39] and the references therein; for the error estimates of the finite difference method, we refer to [6, 30, 49]; and for the error bound of the finite element method, we refer to [2,34, 37. However, few numerical methods have been proposed and/or analyzed for the LogSE due to the singularity at the origin of the logarithmic nonlinearity.

In order to avoid (numerical) blow-up and/or to suppress round-off error due to the logarithmic nonlinearity in the LogSE, a regularized logarithmic Schrödinger equation (RLogSE) with a small regularized parameter $0<\varepsilon \ll 1$ was introduced in 7$]$ as

$$
\left\{\begin{array}{l}
i \partial_{t} u^{\varepsilon}(\mathbf{x}, t)+\Delta u^{\varepsilon}(\mathbf{x}, t)=\lambda u^{\varepsilon}(\mathbf{x}, t) \ln \left(\varepsilon+\left|u^{\varepsilon}(\mathbf{x}, t)\right|\right)^{2}, \quad \mathbf{x} \in \Omega, \quad t>0, \\
u^{\varepsilon}(\mathbf{x}, 0)=u_{0}(\mathbf{x}), \quad \mathbf{x} \in \Omega .
\end{array}\right.
$$

For any fixed $0<\varepsilon \ll 1$, the nonlinearity is now locally Lipschitz continuous (the singularity at the origin disappears). Remarkably enough, 1.12 enjoys similar conservations as the original model (1.1), i.e., the mass (1.2) and momentum (1.3) as well as the regularized energy defined as

$$
\begin{gathered}
E^{\varepsilon}\left(u^{\varepsilon}(\cdot, t)\right)=\int_{\Omega}\left[\left|\nabla u^{\varepsilon}(\mathbf{x}, t)\right|^{2}+2 \lambda \varepsilon\left|u^{\varepsilon}(\mathbf{x}, t)\right|+\lambda\left|u^{\varepsilon}(\mathbf{x}, t)\right|^{2} \ln \left(\varepsilon+\left|u^{\varepsilon}(\mathbf{x}, t)\right|\right)^{2}\right. \\
\left.-\lambda \varepsilon^{2} \ln \left(1+\left|u^{\varepsilon}(\mathbf{x}, t)\right| / \varepsilon\right)^{2}\right] d \mathbf{x} \equiv E^{\varepsilon}\left(u_{0}\right), \quad t \geq 0 .
\end{gathered}
$$


The regularized model 1.12 was proven to approximate the $\operatorname{LogSE}(1.1)$ linearly in $\varepsilon$ for bounded $\Omega$, i.e.,

$$
\sup _{t \in[0, T]}\left\|u^{\varepsilon}(t)-u(t)\right\|_{L^{2}(\Omega)}=O(\varepsilon), \quad \forall T>0,
$$

and with an error $O\left(\varepsilon^{\frac{4}{4+d}}\right)$ in the case of the whole space $\Omega=\mathbb{R}^{d}$, provided that the first two momenta of $u_{0}$ belong to $L^{2}\left(\mathbb{R}^{d}\right)$ 7). In addition, $E^{\varepsilon}\left(u^{\varepsilon}\right)=E(u)+O(\varepsilon)$. Therefore, it is sensible to analyze various numerical methods associated to 1.12 , provided that we have as precise as possible a control of the dependence of the various constants upon $\varepsilon$. Then by using the triangle inequality, we can obtain error estimates of different numerical methods for the LogSE 1.1.

Very recently, a semi-implicit finite difference method was proposed and analyzed for 1.12 and thus for (1.1) 7]. As we know, there are many efficient and accurate numerical methods for the nonlinear Schrödinger equation (1.10) such as the time-splitting spectral method [8,9, 12, 16, 18, 26, 27, 36, 39 and the conservative Crank-Nicolson finite difference (CNFD) method [6. 10]. The main aim of this paper is to present and analyze the time-splitting spectral method and the CNFD method (1.12) and thus for (1.1). We can establish rigorous error estimates for the Lie-Trotter splitting under a much weaker constraint on the time step $\tau$, $\tau \lesssim 1 /\left(\ln \left(\varepsilon^{-1}\right)\right)^{2}$, instead of $\tau \lesssim \sqrt{\varepsilon} e^{-C T|\ln (\varepsilon)|^{2}}$ for some $C$ independent of $\varepsilon$, which is needed for the semi-implicit finite difference method [7].

The rest of the paper is organized as follows. In Section 2, we propose a regularized LieTrotter splitting method and establish rigorous error estimates. In Section 3, a conservative finite difference method is adapted to the RLogSE. Numerical tests are reported for both methods in term of accuracy under different regularities of the initial data. In addition, the splitting method is applied to study the long time dynamics in Section 4.2 and some very interesting and complicated dynamical phenomena are presented to demonstrate the rich dynamics of the Logarithmic Schrödinger equation including interactions of Gaussons. Throughout the paper, $C$ represents a generic constant independent of $\varepsilon, \tau$ and the function $u$, and $C(c)$ means that $C$ depends on $c$.

\section{A regularized Lie-Trotter Splitting method}

In this section, we study the approximation property of a semi-discretization in time for solving the regularized model $\sqrt{1.12}$ for $d=1,2,3$. The numerical integrator we consider is a Lie-Trotter splitting method $25,27,42]$. For simplicity of notation, we set $\lambda=1$.

2.1. Lie-Trotter splitting method. The operator splitting methods for the time integration of 1.12 are based on the splitting

$$
\partial_{t} u^{\varepsilon}=A\left(u^{\varepsilon}\right)+B\left(u^{\varepsilon}\right)
$$

where

$$
A\left(u^{\varepsilon}\right)=i \Delta u^{\varepsilon}, \quad B\left(u^{\varepsilon}\right)=-i \varphi^{\varepsilon}\left(u^{\varepsilon}\right), \quad \varphi^{\varepsilon}(z)=z \ln (\varepsilon+|z|)^{2},
$$

and the solution of the subproblems

$$
\left\{\begin{array}{l}
i \partial_{t} v(\mathbf{x}, t)=-\Delta v(\mathbf{x}, t), \quad \mathbf{x} \in \Omega, \quad t>0, \\
v(\mathbf{x}, 0)=v_{0}(\mathbf{x})
\end{array}\right.
$$




$$
\left\{\begin{array}{l}
i \partial_{t} \omega(\mathbf{x}, t)=\varphi^{\varepsilon}(\omega(\mathbf{x}, t)), \quad \mathbf{x} \in \Omega, \quad t>0 \\
\omega(\mathbf{x}, 0)=\omega_{0}(\mathbf{x})
\end{array}\right.
$$

where $\Omega=\mathbb{R}^{d}$ or $\Omega \subset \mathbb{R}^{d}$ is a bounded domain with homogeneous Dirichlet or periodic boundary condition on the boundary. The associated evolution operators are given by

$$
v(\cdot, t)=\Phi_{A}^{t}\left(v_{0}\right)=e^{i t \Delta} v_{0}, \quad \omega(\cdot, t)=\Phi_{B}^{t}\left(\omega_{0}\right)=\omega_{0} e^{-i t \ln \left(\varepsilon+\left|\omega_{0}\right|\right)^{2}}, \quad t \geq 0 .
$$

Regarding the exact flow $\Phi_{A}^{t}$ and $\Phi_{B}^{t}$, we have the following properties.

Lemma 2.1. For 2.1), we have the isometry relation

$$
\left\|\Phi_{A}^{t}\left(v_{0}\right)\right\|_{H^{s}(\Omega)}=\left\|v_{0}\right\|_{H^{s}(\Omega)}, \quad s \in \mathbb{N}, \quad t \geq 0 .
$$

For 2.2 , if $\omega_{0} \in H^{2}(\Omega)$, then

$$
\begin{aligned}
& \left\|\Phi_{B}^{t}\left(\omega_{0}\right)\right\|_{L^{2}(\Omega)}=\left\|\omega_{0}\right\|_{L^{2}(\Omega)}, \quad\left\|\nabla \Phi_{B}^{t}\left(\omega_{0}\right)\right\|_{L^{2}(\Omega)} \leq(1+2 t)\left\|\nabla \omega_{0}\right\|_{L^{2}(\Omega)}, \\
& \left\|\Phi_{B}^{t}\left(\omega_{0}\right)\right\|_{H^{2}(\Omega)} \leq C\left(\left\|\omega_{0}\right\|_{H^{2}(\Omega)}\right)\left(1+t+t^{2}\right) / \varepsilon
\end{aligned}
$$

Proof. By direct calculation, we get

$$
\nabla \Phi_{B}^{t}\left(\omega_{0}\right)=e^{-i t \ln \left(\varepsilon+\left|\omega_{0}\right|\right)^{2}}\left[\nabla \omega_{0}-\frac{2 i t \omega_{0}}{\varepsilon+\left|\omega_{0}\right|} \nabla\left|\omega_{0}\right|\right] \text {, with } \nabla\left|\omega_{0}\right|=\frac{\omega_{0} \nabla \overline{\omega_{0}}+\overline{\omega_{0}} \nabla \omega_{0}}{2\left|\omega_{0}\right|},
$$

which gives immediately, since $|\nabla| \omega_{0}|| \leq\left|\nabla \omega_{0}\right|$,

$$
\left\|\nabla \Phi_{B}^{t}\left(\omega_{0}\right)\right\|_{L^{2}(\Omega)} \leq(1+2 t)\left\|\nabla \omega_{0}\right\|_{L^{2}(\Omega)} .
$$

Noticing that

$$
\begin{aligned}
\frac{\partial^{2} \Phi_{B}^{t}\left(\omega_{0}\right)}{\partial x_{k} \partial x_{j}}= & \frac{\partial^{2} \omega_{0}}{\partial x_{k} \partial x_{j}}-\frac{2 i t}{\varepsilon+\left|\omega_{0}\right|}\left(\frac{\partial \omega_{0}}{\partial x_{k}} \frac{\partial\left|\omega_{0}\right|}{\partial x_{j}}+\frac{\partial \omega_{0}}{\partial x_{j}} \frac{\partial\left|\omega_{0}\right|}{\partial x_{k}}+\omega_{0} \frac{\partial^{2}\left|\omega_{0}\right|}{\partial x_{k} \partial x_{j}}\right) \\
& +\frac{2 i t-4 t^{2}}{\left(\varepsilon+\left|\omega_{0}\right|\right)^{2}} \frac{\partial\left|\omega_{0}\right|}{\partial x_{j}} \frac{\partial\left|\omega_{0}\right|}{\partial x_{k}} \omega_{0},
\end{aligned}
$$

where

$$
\begin{aligned}
\left|\frac{\partial^{2}\left|\omega_{0}\right|}{\partial x_{k} \partial x_{j}}\right| & =\left|\frac{1}{\left|\omega_{0}\right|} \operatorname{Re}\left(\omega_{0} \frac{\partial^{2} \overline{\omega_{0}}}{\partial x_{k} \partial x_{j}}+\frac{\partial \omega_{0}}{\partial x_{j}} \frac{\partial \overline{\omega_{0}}}{\partial x_{k}}\right)-\frac{1}{\left|\omega_{0}\right|^{2}} \frac{\partial\left|\omega_{0}\right|}{\partial x_{k}} \operatorname{Re}\left(\omega_{0} \frac{\partial \overline{\omega_{0}}}{\partial x_{j}}\right)\right| \\
& \leq\left|\frac{\partial^{2} \omega_{0}}{\partial x_{k} \partial x_{j}}\right|+\frac{2}{\left|\omega_{0}\right|}\left|\frac{\partial \omega_{0}}{\partial x_{j}}\right|\left|\frac{\partial \omega_{0}}{\partial x_{k}}\right|
\end{aligned}
$$

this yields that

$$
\begin{aligned}
\left|\frac{\partial^{2} \Phi_{B}^{t}\left(\omega_{0}\right)}{\partial x_{k} \partial x_{j}}\right| & \leq(1+2 t)\left|\frac{\partial^{2} \omega_{0}}{\partial x_{k} \partial x_{j}}\right|+\frac{10 t+4 t^{2}}{\varepsilon+\left|\omega_{0}\right|}\left|\frac{\partial \omega_{0}}{\partial x_{j}}\right|\left|\frac{\partial \omega_{0}}{\partial x_{k}}\right| \\
& \leq(1+2 t)\left|\frac{\partial^{2} \omega_{0}}{\partial x_{k} \partial x_{j}}\right|+\frac{5 t+2 t^{2}}{\varepsilon}\left(\left|\frac{\partial \omega_{0}}{\partial x_{j}}\right|^{2}+\left|\frac{\partial \omega_{0}}{\partial x_{k}}\right|^{2}\right) .
\end{aligned}
$$

Thus

$$
\left\|\frac{\partial^{2} \Phi_{B}^{t}\left(\omega_{0}\right)}{\partial x_{k} \partial x_{j}}\right\|_{L^{2}(\Omega)} \leq(1+2 t)\left\|\frac{\partial^{2} \omega_{0}}{\partial x_{k} \partial x_{j}}\right\|_{L^{2}(\Omega)}+\frac{5 t+2 t^{2}}{\varepsilon}\left(\left\|\frac{\partial \omega_{0}}{\partial x_{j}}\right\|_{L^{4}(\Omega)}^{2}+\left\|\frac{\partial \omega_{0}}{\partial x_{k}}\right\|_{L^{4}(\Omega)}^{2}\right),
$$

which completes the proof by recalling that $H^{2}(\Omega) \hookrightarrow W^{1,4}(\Omega)$, since $d \leq 3$. 
We consider the Lie-Trotter splitting

$$
u^{\varepsilon, n+1}=\Phi^{\tau}\left(u^{\varepsilon, n}\right)=\Phi_{A}^{\tau}\left(\Phi_{B}^{\tau}\left(u^{\varepsilon, n}\right)\right), \quad u^{\varepsilon, 0}=u_{0},
$$

for a time step $\tau>0$. Thus for $u^{\varepsilon, n} \in H^{1}(\Omega)$, it follows from the isometry property that the splitting method conserves the mass

$$
\left\|u^{\varepsilon, n+1}\right\|_{L^{2}(\Omega)}=\left\|u^{\varepsilon, n}\right\|_{L^{2}(\Omega)} \equiv\left\|u^{\varepsilon, 0}\right\|_{L^{2}(\Omega)}=\left\|u_{0}\right\|_{L^{2}(\Omega)}, \quad n \geq 0,
$$

and furthermore $(2.5)$ gives $u^{\varepsilon, n} \in H^{1}(\Omega)$ with

$$
\left\|u^{\varepsilon, n+1}\right\|_{H^{1}(\Omega)} \leq(1+2 \tau)\left\|u^{\varepsilon, n}\right\|_{H^{1}(\Omega)} \leq e^{2 n \tau}\left\|u_{0}\right\|_{H^{1}(\Omega)}, \quad n \geq 0 .
$$

2.2. Error estimates. In this section, we carry out the error analysis of the Lie-Trotter splitting (2.6).

Theorem 2.2. Let $T>0$. Assume that the solution of $(1.12)$ satisfies $u^{\varepsilon} \in L^{\infty}\left(0, T ; H^{1}(\Omega)\right)$ for $d=1$ or $u^{\varepsilon} \in L^{\infty}\left(0, T ; H^{2}(\Omega)\right)$ for $d=2,3$. There exists $\varepsilon_{0}>0$ depending on $\left\|u^{\varepsilon}\right\|_{L^{\infty}\left(0, T ; H^{1}(\Omega)\right)}$ for $d=1$, and $\left\|u^{\varepsilon}\right\|_{L^{\infty}\left(0, T ; H^{2}(\Omega)\right)}$ for $d=2,3$, such that when $\varepsilon \leq \varepsilon_{0}$ and $n \tau \leq T$, we have

$$
\left\|u^{\varepsilon, n}-u^{\varepsilon}\left(t_{n}\right)\right\|_{L^{2}(\Omega)} \leq C\left(T,\left\|u^{\varepsilon}\right\|_{L^{\infty}\left([0, T] ; H^{1}(\Omega)\right)}\right) \ln \left(\varepsilon^{-1}\right) \tau^{1 / 2},
$$

where $C(\cdot, \cdot)$ is independent of $\varepsilon>0$.

Remark 2.3. As established in [7, Theorem 2.2], for an arbitrarily large fixed $T>0$, the above assumptions are satisfied as soon as $u_{0} \in H^{1}(\Omega)$ if $d=1$, and $u_{0} \in H^{2}(\Omega)$ if $d=2,3$. More precisely, for $k=1,2$,

$$
\sup _{t \in[0, T]}\left\|u^{\varepsilon}(t)\right\|_{H^{k}(\Omega)} \leq C\left(\left\|u_{0}\right\|_{H^{k}(\Omega)}\right),
$$

for a constant $C$ depending on $\left\|u_{0}\right\|_{H^{k}(\Omega)}$, but not on $0<\varepsilon \ll 1$.

The above result provides a convergence of order $1 / 2$, with a constant mildly singular in $\varepsilon$ (a logarithm). We will see in Remark 2.7 that it is possible to establish the convergence of order 1, which is rather natural for a Lie-Trotter scheme, but the price to pay is a much more singular dependence with respect to $\varepsilon$. And numerical observations show that the convergence rate degenerates to $1 / 2$ for solutions belonging to $H^{1}$ in $1 \mathrm{D}$ (cf. first figure in Fig. 4.3).

Before giving the proof, we introduce the following lemma, which is a variant of 20 , Lemma 9.3.5], established in [7.

Lemma 2.4. For any $z_{1}, z_{2} \in \mathbb{C}$, we have

$$
\left|\operatorname{Im}\left(\left(\varphi^{\varepsilon}\left(z_{1}\right)-\varphi^{\varepsilon}\left(z_{2}\right)\right)\left(\overline{z_{1}}-\overline{z_{2}}\right)\right)\right| \leq 2\left|z_{1}-z_{2}\right|^{2} .
$$

Lemma 2.5 (Local error). Assume $u_{0} \in H^{1}(\Omega)$ for $d=1$ or $u_{0} \in H^{2}(\Omega)$ for $d=2,3$. Let $\Psi^{t}$ denote the exact flow of $(1.12)$, i.e., $u^{\varepsilon}(t)=\Psi^{t}\left(u_{0}\right)$. Then for $\tau \leq 1$, there exists $\varepsilon_{0}>0$ depending on $\left\|u_{0}\right\|_{H^{1}(\Omega)}$ for $d=1$ and $\left\|u_{0}\right\|_{H^{2}(\Omega)}$ for $d=2,3$ such that when $\varepsilon \leq \varepsilon_{0}$, we have

$$
\left\|\Psi^{\tau}\left(u_{0}\right)-\Phi^{\tau}\left(u_{0}\right)\right\|_{L^{2}(\Omega)} \leq C\left\|u_{0}\right\|_{H^{1}(\Omega)} \ln \left(\varepsilon^{-1}\right) \tau^{3 / 2} .
$$

Proof. It can be obtained from the definition that

$$
\begin{aligned}
& i \partial_{t}\left(\Psi^{t} u_{0}\right)+\Delta\left(\Psi^{t} u_{0}\right)=\varphi^{\varepsilon}\left(\Psi^{t} u_{0}\right) \\
& i \partial_{t}\left(\Phi^{t} u_{0}\right)+\Delta\left(\Phi^{t} u_{0}\right)=\Phi_{A}^{t}\left(\varphi^{\varepsilon}\left(\Phi_{B}^{t} u_{0}\right)\right) .
\end{aligned}
$$


Denoting $\mathcal{E}^{t} u_{0}=\Psi^{t} u_{0}-\Phi^{t} u_{0}$, we have

$$
i \partial_{t}\left(\mathcal{E}^{t} u_{0}\right)+\Delta\left(\mathcal{E}^{t} u_{0}\right)=\varphi^{\varepsilon}\left(\Psi^{t} u_{0}\right)-\Phi_{A}^{t}\left(\varphi^{\varepsilon}\left(\Phi_{B}^{t} u_{0}\right)\right)
$$

Denote by

$$
(f, g)=\int_{\Omega} f \bar{g} d \mathbf{x}
$$

the $L^{2}$ inner product. Multiplying $(2.8)$ by $\overline{\mathcal{E}^{t} u_{0}}$, integrating in space and taking the imaginary part, the term corresponding to the Laplacian vanishes (in the case with a boundary, we use the Dirichlet boundary condition or periodic boundary condition), and Lemma 2.4 yields

$$
\begin{aligned}
\frac{1}{2} \frac{d}{d t}\left\|\mathcal{E}^{t} u_{0}\right\|_{L^{2}(\Omega)}^{2} & =\operatorname{Im}\left(\varphi^{\varepsilon}\left(\Psi^{t} u_{0}\right)-\Phi_{A}^{t}\left(\varphi^{\varepsilon}\left(\Phi_{B}^{t} u_{0}\right)\right), \mathcal{E}^{t} u_{0}\right) \\
& =\operatorname{Im}\left[\left(\varphi^{\varepsilon}\left(\Psi^{t} u_{0}\right)-\varphi^{\varepsilon}\left(\Phi^{t} u_{0}\right), \mathcal{E}^{t} u_{0}\right)+\left(\varphi^{\varepsilon}\left(\Phi^{t} u_{0}\right)-\Phi_{A}^{t}\left(\varphi^{\varepsilon}\left(\Phi_{B}^{t} u_{0}\right)\right), \mathcal{E}^{t} u_{0}\right)\right] \\
& \leq 2\left\|\mathcal{E}^{t} u_{0}\right\|_{L^{2}(\Omega)}^{2}+\left\|\varphi^{\varepsilon}\left(\Phi^{t} u_{0}\right)-\Phi_{A}^{t}\left(\varphi^{\varepsilon}\left(\Phi_{B}^{t} u_{0}\right)\right)\right\|_{L^{2}(\Omega)}\left\|\mathcal{E}^{t} u_{0}\right\|_{L^{2}(\Omega)},
\end{aligned}
$$

which implies

$$
\begin{aligned}
\frac{d}{d t}\left\|\mathcal{E}^{t} u_{0}\right\|_{L^{2}(\Omega)} \leq & 2\left\|\mathcal{E}^{t} u_{0}\right\|_{L^{2}(\Omega)}+\left\|\varphi^{\varepsilon}\left(\Phi^{t} u_{0}\right)-\Phi_{A}^{t}\left(\varphi^{\varepsilon}\left(\Phi_{B}^{t} u_{0}\right)\right)\right\|_{L^{2}(\Omega)} \\
\leq & 2\left\|\mathcal{E}^{t} u_{0}\right\|_{L^{2}(\Omega)}+\left\|\varphi^{\varepsilon}\left(\Phi^{t} u_{0}\right)-\varphi^{\varepsilon}\left(\Phi_{B}^{t} u_{0}\right)\right\|_{L^{2}(\Omega)} \\
& +\left\|\varphi^{\varepsilon}\left(\Phi_{B}^{t} u_{0}\right)-\Phi_{A}^{t}\left(\varphi^{\varepsilon}\left(\Phi_{B}^{t} u_{0}\right)\right)\right\|_{L^{2}(\Omega)}
\end{aligned}
$$

If $\Omega=\mathbb{R}^{d}$, for any $f \in H^{1}(\Omega)$, we compute

$$
\begin{aligned}
\left\|f-\Phi_{A}^{t} f\right\|_{L^{2}(\Omega)} & =\left\|\left(1-e^{-i t|\xi|^{2}}\right) \widehat{f}(\xi)\right\|_{L^{2}(\Omega)}=2\left\|\sin \left(t|\xi|^{2} / 2\right) \widehat{f}(\xi)\right\|_{L^{2}(\Omega)} \\
& \leq \sqrt{2 t}\||\xi| \widehat{f}(\xi)\|_{L^{2}(\Omega)} \leq \sqrt{2 t}\|f\|_{H^{1}(\Omega)}
\end{aligned}
$$

When $\Omega$ is a bounded domain, 2.10 can be similarly obtained via the discrete Fourier transform. At this stage, one could argue that the above estimate can be improved, by removing the square root, the price to pay being an $H^{2}$-norm instead of an $H^{1}$-norm. It turns out that this approach eventually yields an extra $1 / \varepsilon$ factor in the error estimate, which we want to avoid here; see Remark 2.7. Recalling that $\varphi^{\varepsilon}\left(\Phi_{B}^{t} u_{0}\right)=u_{0} \ln \left(\varepsilon+\left|u_{0}\right|\right)^{2} e^{-i t \ln \left(\varepsilon+\left|u_{0}\right|\right)^{2}}$, we compute

$$
\nabla \varphi^{\varepsilon}\left(\Phi_{B}^{t} u_{0}\right)=e^{-i t \ln \left(\varepsilon+\left|u_{0}\right|\right)^{2}}\left[\nabla u_{0} \ln \left(\varepsilon+\left|u_{0}\right|\right)^{2}+\frac{2 u_{0} \nabla\left|u_{0}\right|}{\varepsilon+\left|u_{0}\right|}\left(1-i t \ln \left(\varepsilon+\left|u_{0}\right|\right)^{2}\right)\right],
$$

which yields

$$
\left|\nabla \varphi^{\varepsilon}\left(\Phi_{B}^{t} u_{0}\right)\right| \leq 2\left|\nabla u_{0}\right|\left(1+(1+2 t) \max \left\{|\ln (\varepsilon)|,\left|\ln \left(\varepsilon+\left\|u_{0}\right\|_{L^{\infty}(\Omega)}\right)\right|\right\}\right) .
$$

This implies

$$
\left\|\varphi^{\varepsilon}\left(\Phi_{B}^{t} u_{0}\right)\right\|_{H^{1}(\Omega)} \leq C \ln \left(\varepsilon^{-1}\right)(1+t)\left\|u_{0}\right\|_{H^{1}(\Omega)},
$$

when $\varepsilon \lesssim 1 /\left\|u_{0}\right\|_{L^{\infty}(\Omega)}$. To check this property, we recall that $H^{1}(\Omega) \hookrightarrow L^{\infty}(\Omega)$ for $d=1$, and $H^{2}(\Omega) \hookrightarrow L^{\infty}(\Omega)$ for $d=2,3$. It follows from $(2.4)$ and $(2.5)$ that

$$
\left\|\Phi^{t} u_{0}\right\|_{H^{2}(\Omega)}=\left\|\Phi_{B}^{t} u_{0}\right\|_{H^{2}(\Omega)} \leq C\left(\left\|u_{0}\right\|_{H^{2}(\Omega)}\right)\left(1+t+t^{2}\right) / \varepsilon
$$


Hence by Sobolev imbedding, when $t \leq 1$, we have $\left\|\Phi^{t} u_{0}\right\|_{L^{\infty}(\Omega)},\left\|\Phi_{B}^{t} u_{0}\right\|_{L^{\infty}(\Omega)} \leq \frac{C\left(\left\|u_{0}\right\|_{H^{2}(\Omega)}\right)}{\varepsilon}$ for $d=2,3$ and $\left\|\Phi^{t} u_{0}\right\|_{L^{\infty}(\Omega)},\left\|\Phi_{B}^{t} u_{0}\right\|_{L^{\infty}(\Omega)} \leq C\left\|u_{0}\right\|_{H^{1}(\Omega)}$ for $d=1$. In particular, the property $\varepsilon \lesssim 1 /\left\|u_{0}\right\|_{L^{\infty}(\Omega)}$ is always satisfied.

Hence we have

$$
\begin{aligned}
\left\|\varphi^{\varepsilon}\left(\Phi_{B}^{t} u_{0}\right)-\Phi_{A}^{t}\left(\varphi^{\varepsilon}\left(\Phi_{B}^{t} u_{0}\right)\right)\right\|_{L^{2}(\Omega)} & \leq \sqrt{2 t}\left\|\varphi^{\varepsilon}\left(\Phi_{B}^{t} u_{0}\right)\right\|_{H^{1}(\Omega)} \\
& \leq C \ln \left(\varepsilon^{-1}\right) \sqrt{t}(1+t)\left\|u_{0}\right\|_{H^{1}(\Omega)}
\end{aligned}
$$

for $\varepsilon \leq \varepsilon_{1}$, with $\varepsilon_{1}$ depending on $\left\|u_{0}\right\|_{H^{1}(\Omega)}$ for $d=1$ and $\left\|u_{0}\right\|_{H^{2}(\Omega)}$ for $d=2,3$. Next we claim that for $v(\mathbf{x}), w(\mathbf{x})$ satisfying $|v(\mathbf{x})|,|w(\mathbf{x})| \leq C_{1} / \varepsilon$, it can be established that

$$
\left|\varphi^{\varepsilon}(v(\mathbf{x}))-\varphi^{\varepsilon}(w(\mathbf{x}))\right| \leq C \ln \left(\varepsilon^{-1}\right)|v(\mathbf{x})-w(\mathbf{x})|,
$$

when $\varepsilon$ is sufficiently small. Assuming, for example, $0 \leq|w(\mathbf{x})| \leq|v(\mathbf{x})|$, then

$$
\begin{aligned}
\left|\varphi^{\varepsilon}(v(\mathbf{x}))-\varphi^{\varepsilon}(w(\mathbf{x}))\right| & =2\left|(v(\mathbf{x})-w(\mathbf{x})) \ln (\varepsilon+|v(\mathbf{x})|)+w(\mathbf{x}) \ln \left(1+\frac{|v(\mathbf{x})|-|w(\mathbf{x})|}{\varepsilon+|w(\mathbf{x})|}\right)\right| \\
& \leq 2|v(\mathbf{x})-w(\mathbf{x})||\ln (\varepsilon+|v(\mathbf{x})|)|+\frac{2|w(\mathbf{x})|}{\varepsilon+|w(\mathbf{x})|}|v(\mathbf{x})-w(\mathbf{x})| \\
& \leq C \ln \left(\varepsilon^{-1}\right)|v(\mathbf{x})-w(\mathbf{x})|,
\end{aligned}
$$

when $\varepsilon \leq \min \left(C_{1}, 1\right)$. Thus, we obtain

$$
\begin{aligned}
\left\|\varphi^{\varepsilon}\left(\Phi^{t} u_{0}\right)-\varphi^{\varepsilon}\left(\Phi_{B}^{t} u_{0}\right)\right\|_{L^{2}(\Omega)} & \leq C \ln \left(\varepsilon^{-1}\right)\left\|\Phi^{t} u_{0}-\Phi_{B}^{t} u_{0}\right\|_{L^{2}(\Omega)} \\
& \leq C \ln \left(\varepsilon^{-1}\right) \sqrt{2 t}\left\|\Phi_{B}^{t} u_{0}\right\|_{H^{1}(\Omega)} \\
& \leq C \ln \left(\varepsilon^{-1}\right) \sqrt{2 t}(1+2 t)\left\|u_{0}\right\|_{H^{1}(\Omega)},
\end{aligned}
$$

when $\varepsilon \leq \varepsilon_{2}$ with $\varepsilon_{2}$ depending on $\left\|u_{0}\right\|_{H^{2}(\Omega)}$ for $d=2,3$ and $\left\|u_{0}\right\|_{H^{1}(\Omega)}$ for $d=1$. Combining (2.9), 2.11) and (2.13), we get

$$
\frac{d}{d t}\left\|\mathcal{E}^{t} u_{0}\right\|_{L^{2}(\Omega)} \leq 2\left\|\mathcal{E}^{t} u_{0}\right\|_{L^{2}(\Omega)}+C \ln \left(\varepsilon^{-1}\right) \sqrt{t}(1+t)\left\|u_{0}\right\|_{H^{1}(\Omega)}
$$

Applying the Gronwall's inequality, when $\tau \leq 1$, we have

$$
\left\|\mathcal{E}^{\tau} u_{0}\right\|_{L^{2}(\Omega)} \leq C \ln \left(\varepsilon^{-1}\right) \sqrt{\tau}(1+\tau)\left(e^{2 \tau}-1\right)\left\|u_{0}\right\|_{H^{1}(\Omega)} \leq C \ln \left(\varepsilon^{-1}\right) \tau^{3 / 2}\left\|u_{0}\right\|_{H^{1}(\Omega)},
$$

when $\varepsilon \leq \varepsilon_{0}=\min \left\{\varepsilon_{1}, \varepsilon_{2}\right\}$ depending on $\left\|u_{0}\right\|_{H^{1}(\Omega)}$ for $d=1$ and $\left\|u_{0}\right\|_{H^{2}(\Omega)}$ for $d=2,3$.

Furthermore, we also need the following lemma concerning on the stability property.

Lemma 2.6 (Stability). Let $f, g \in L^{2}(\Omega)$. Then for all $\tau>0$, we have

$$
\left\|\Phi^{\tau}(f)-\Phi^{\tau}(g)\right\|_{L^{2}(\Omega)} \leq(1+2 \tau)\|f-g\|_{L^{2}(\Omega)} .
$$

Proof. Noticing that $\Phi_{A}^{\tau}$ is a linear isometry on $H^{s}(\Omega)$, we obtain that

$$
\left\|\Phi^{\tau}(f)-\Phi^{\tau}(g)\right\|_{L^{2}(\Omega)}=\left\|\Phi_{B}^{\tau}(f)-\Phi_{B}^{\tau}(g)\right\|_{L^{2}(\Omega)} .
$$

We claim that for any $\mathbf{x} \in \Omega$, we have

$$
\left|\Phi_{B}^{\tau}(f)(\mathbf{x})-\Phi_{B}^{\tau}(g)(\mathbf{x})\right| \leq(1+2 \tau)|f(\mathbf{x})-g(\mathbf{x})| .
$$


Assuming, for example, $|f(\mathbf{x})| \leq|g(\mathbf{x})|$, then by inserting a term $f(\mathbf{x}) e^{-i \tau \ln (\varepsilon+|g(\mathbf{x})|)^{2}}$, we can get that

$$
\begin{aligned}
\left|\Phi_{B}^{\tau}(f)(\mathbf{x})-\Phi_{B}^{\tau}(g)(\mathbf{x})\right| & =\left|f(\mathbf{x}) e^{-i \tau \ln (\varepsilon+|f(\mathbf{x})|)^{2}}-g(\mathbf{x}) e^{-i \tau \ln (\varepsilon+|g(\mathbf{x})|)^{2}}\right| \\
& =\mid f(\mathbf{x})-g(\mathbf{x})+f(\mathbf{x})\left(e^{2 i \tau \ln \left(\frac{\varepsilon+|g(\mathbf{x})|}{\varepsilon+|f(\mathbf{x})|}-1\right) \mid}\right. \\
& \leq|f(\mathbf{x})-g(\mathbf{x})|+2|f(\mathbf{x})|\left|\sin \left(\tau \ln \left(\frac{\varepsilon+|g(\mathbf{x})|}{\varepsilon+|f(\mathbf{x})|}\right)\right)\right| \\
& \leq|f(\mathbf{x})-g(\mathbf{x})|+2 \tau|f(\mathbf{x})| \ln \left(1+\frac{|g(\mathbf{x})|-|f(\mathbf{x})|}{\varepsilon+|f(\mathbf{x})|}\right) \\
& \leq(1+2 \tau)|f(\mathbf{x})-g(\mathbf{x})| .
\end{aligned}
$$

When $|f(\mathbf{x})| \geq|g(\mathbf{x})|$, the same inequality is obtained by exchanging $f$ and $g$ in the above computation. Thus the proof is completed.

Proof of Theorem 2.2. It can be easily concluded from 2.7 that $u^{\varepsilon, n} \in H^{1}(\Omega)$ and $\left\|u^{\varepsilon, n}\right\|_{H^{1}(\Omega)} \leq$ $e^{2 T}\left\|u_{0}\right\|_{H^{1}(\Omega)}$. The triangle inequality, (2.7), Lemmas 2.5 and 2.6 yield

$$
\begin{aligned}
\left\|u^{\varepsilon, n}-u^{\varepsilon}\left(t_{n}\right)\right\|_{L^{2}(\Omega)} \\
=\left\|\Phi^{\tau}\left(u^{\varepsilon, n-1}\right)-\Psi^{\tau}\left(u^{\varepsilon}\left(t_{n-1}\right)\right)\right\|_{L^{2}(\Omega)} \\
\leq\left\|\Phi^{\tau}\left(u^{\varepsilon, n-1}\right)-\Phi^{\tau}\left(u^{\varepsilon}\left(t_{n-1}\right)\right)\right\|_{L^{2}(\Omega)}+\left\|\Phi^{\tau}\left(u^{\varepsilon}\left(t_{n-1}\right)\right)-\Psi^{\tau}\left(u^{\varepsilon}\left(t_{n-1}\right)\right)\right\|_{L^{2}(\Omega)} \\
\leq(1+2 \tau)\left\|u^{\varepsilon, n-1}-u^{\varepsilon}\left(t_{n-1}\right)\right\|_{L^{2}(\Omega)}+\left\|\mathcal{E}^{\tau}\left(u^{\varepsilon}\left(t_{n-1}\right)\right)\right\|_{L^{2}(\Omega)} \\
\leq(1+2 \tau)\left\|u^{\varepsilon, n-1}-u^{\varepsilon}\left(t_{n-1}\right)\right\|_{L^{2}(\Omega)}+C\left\|u^{\varepsilon}\left(t_{n-1}\right)\right\|_{H^{1}(\Omega)} \ln \left(\varepsilon^{-1}\right) \tau^{3 / 2} \\
\leq C\left\|u^{\varepsilon}\right\|_{L^{\infty}\left(0, T ; H^{1}(\Omega)\right)} \ln \left(\varepsilon^{-1}\right) \tau^{3 / 2}(1+1+2 \tau)+(1+2 \tau)^{2}\left\|u^{\varepsilon, n-2}-u^{\varepsilon}\left(t_{n-2}\right)\right\|_{L^{2}(\Omega)} \\
\leq \\
\leq \\
\leq \\
\quad+\left(1+u^{\varepsilon}\left\|_{L^{\infty}\left(0, T ; H^{1}(\Omega)\right)} \ln \left(\varepsilon^{-1}\right) \tau^{3 / 2}\left[1+(1+2 \tau)+\cdots+(1+2 \tau)^{n-1}\right]\right\| u^{\varepsilon, 0}-u_{0} \|_{L^{2}(\Omega)}\right. \\
\leq C\left\|u^{\varepsilon}\right\|_{L^{\infty}\left(0, T ; H^{1}(\Omega)\right)} \ln \left(\varepsilon^{-1}\right) \tau^{1 / 2}(1+2 \tau)^{n} \\
\leq C\left\|u^{\varepsilon}\right\|_{L^{\infty}\left(0, T ; H^{1}(\Omega)\right)} e^{2 T} \ln \left(\varepsilon^{-1}\right) \tau^{1 / 2},
\end{aligned}
$$

where we have used $u^{\varepsilon, 0}=u_{0}$, see (2.6). This completes the proof.

Remark 2.7. Noticing that for any $f \in H^{2}(\Omega)$ and $t \geq 0$, we have 12]

$$
\left\|f-\Phi_{A}^{t} f\right\|_{L^{2}(\Omega)} \leq t\|f\|_{H^{2}(\Omega)},
$$

and by tedious calculation, one can get that

$$
\left\|\varphi^{\varepsilon}(f)\right\|_{H^{2}(\Omega)} \leq C \ln \left(\varepsilon^{-1}\right)\|f\|_{H^{2}(\Omega)}+C \varepsilon^{-1}\|\nabla f\|_{L^{4}(\Omega)}^{2}
$$


it can be concluded from (2.9) that

$$
\begin{aligned}
\frac{d}{d t}\left\|\mathcal{E}^{t} u_{0}\right\|_{L^{2}(\Omega)} \leq & 2\left\|\mathcal{E}^{t} u_{0}\right\|_{L^{2}(\Omega)}+\left\|\varphi^{\varepsilon}\left(\Phi^{t} u_{0}\right)-\varphi^{\varepsilon}\left(\Phi_{B}^{t} u_{0}\right)\right\|_{L^{2}(\Omega)} \\
& +\left\|\varphi^{\varepsilon}\left(\Phi_{B}^{t} u_{0}\right)-\Phi_{A}^{t}\left(\varphi^{\varepsilon}\left(\Phi_{B}^{t} u_{0}\right)\right)\right\|_{L^{2}(\Omega)} \\
\leq & 2\left\|\mathcal{E}^{t} u_{0}\right\|_{L^{2}(\Omega)}+C \ln \left(\varepsilon^{-1}\right)\left\|\Phi^{t} u_{0}-\Phi_{B}^{t} u_{0}\right\|_{L^{2}(\Omega)}+t\left\|\varphi^{\varepsilon}\left(\Phi_{B}^{t} u_{0}\right)\right\|_{H^{2}(\Omega)} \\
\leq & 2\left\|\mathcal{E}^{t} u_{0}\right\|_{L^{2}(\Omega)}+C t \ln \left(\varepsilon^{-1}\right)\left\|\Phi_{B}^{t} u_{0}\right\|_{H^{2}(\Omega)}+C t \varepsilon^{-1}\left\|\nabla \Phi_{B}^{t} u_{0}\right\|_{L^{4}(\Omega)}^{2} \\
\leq & 2\left\|\mathcal{E}^{t} u_{0}\right\|_{L^{2}(\Omega)}+C\left(\left\|u_{0}\right\|_{H^{2}(\Omega)}\right) \varepsilon^{-1} \ln \left(\varepsilon^{-1}\right) t+C t \varepsilon^{-1}\left\|\nabla u_{0}\right\|_{L^{4}(\Omega)}^{2} \\
\leq & 2\left\|\mathcal{E}^{t} u_{0}\right\|_{L^{2}(\Omega)}+C\left(\left\|u_{0}\right\|_{H^{2}(\Omega)}\right) \varepsilon^{-1} \ln \left(\varepsilon^{-1}\right) t
\end{aligned}
$$

when $\varepsilon$ is sufficiently small. Hence by using similar arguments, we can get that

$$
\left\|u^{\varepsilon, n}-u^{\varepsilon}\left(t_{n}\right)\right\|_{L^{2}(\Omega)} \leq C\left(T,\left\|u^{\varepsilon}\right\|_{L^{\infty}\left([0, T] ; H^{2}(\Omega)\right)}\right) \varepsilon^{-1} \ln \left(\varepsilon^{-1}\right) \tau,
$$

when $\varepsilon \leq c$, where $c>0$ depends on $\left\|u^{\varepsilon}\right\|_{L^{\infty}\left(0, T ; H^{1}(\Omega)\right)}$ for $d=1$ and $\left\|u^{\varepsilon}\right\|_{L^{\infty}\left(0, T ; H^{2}(\Omega)\right)}$ for $d=2,3$. This approach yields a better convergence rate for fixed $\varepsilon>0$, but with a terrible dependence upon $\varepsilon$, as $\varepsilon$ is intended to go to zero to recover the solution of (1.1). Following Theorem 2.2, we get a reasonable numerical approximation of the solution u to (1.1) provided that $\tau \ll 1 /\left(\ln \left(\varepsilon^{-1}\right)\right)^{2}$ and $\varepsilon \ll 1$ (for $u^{\varepsilon}$ to approximate $u$ ), while the above estimate requires the stronger condition $\tau \ll \varepsilon / \ln \left(\varepsilon^{-1}\right)$, and still $\varepsilon \ll 1$.

Remark 2.8. For the other Lie-Trotter splitting

$$
u^{\varepsilon, n+1}=\Phi_{B}^{\tau}\left(\Phi_{A}^{\tau}\left(u^{\varepsilon, n}\right)\right)=\Phi_{A}^{\tau}\left(u^{\varepsilon, n}\right)-i \int_{0}^{\tau} \varphi^{\varepsilon}\left(\Phi_{B}^{s} \Phi_{A}^{\tau}\left(u^{\varepsilon, n}\right)\right) d s,
$$

unfortunately, we cannot get the similar error estimate as in Theorem 2.2, since the proof involves $\left(\varphi^{\varepsilon}\right)^{\prime}$ or even $\left(\varphi^{\varepsilon}\right)^{\prime \prime}$, which yields negative powers of $\varepsilon$ in the local error. In fact, by using the standard arguments via the Lie commutator as in [39], we can get the error bound

$$
\left\|u^{\varepsilon, n}-u^{\varepsilon}\left(t_{n}\right)\right\|_{L^{2}(\Omega)} \leq C\left(T,\left\|u^{\varepsilon}\right\|_{L^{\infty}\left([0, T] ; H^{2}(\Omega)\right)}\right) \varepsilon^{-1} \tau,
$$

when $\varepsilon \leq c$ with $c$ depending on $\left\|u^{\varepsilon}\right\|_{L^{\infty}\left(0, T ; H^{2}(\Omega)\right)}$.

Remark 2.9 (Strang splitting). When considering a Strang splitting,

$$
u^{\varepsilon, n+1}=\Phi_{B}^{\tau / 2}\left(\Phi_{A}^{\tau}\left(\Phi_{B}^{\tau / 2}\left(u^{\varepsilon, n}\right)\right)\right),
$$

or

$$
u^{\varepsilon, n+1}=\Phi_{A}^{\tau / 2}\left(\Phi_{B}^{\tau}\left(\Phi_{A}^{\tau / 2}\left(u^{\varepsilon, n}\right)\right)\right),
$$

one would expect to face similar singular factors as above. It turns out that the analysis is even more intricate than expected, and we could not get any reasonable estimate in that case, that is, improving Theorem 2.2 in terms of order for fixed $\varepsilon$, without (too much) singularity in $\varepsilon$. This can be understood as a remain of the singularity of the logarithm at the origin, yielding too many negative powers of $\varepsilon$ in the case of (1.12). Strang splitting usually provides better error estimates by invoking higher regularity which, in our case, implies extra negative powers of $\varepsilon$. 


\section{A Regularized Crank-Nicolson finite difference method}

In this section, we introduce a conservative Crank-Nicolson finite difference (CNFD) method for solving the regularized model (1.12). For simplicity of notation, we only present the numerical method for the RLogSE (1.12) in 1D, as extensions to higher dimensions are straightforward. When $d=1$, we truncate the RLogSE on a bounded computational interval $\Omega=(a, b)$ with periodic boundary condition (here $|a|$ and $b$ are chosen large enough such that the truncation error is negligible):

$$
\left\{\begin{array}{l}
i \partial_{t} u^{\varepsilon}(x, t)+\partial_{x x} u^{\varepsilon}(x, t)=\lambda u^{\varepsilon}(x, t) \ln \left(\varepsilon+\left|u^{\varepsilon}(x, t)\right|\right)^{2}, \quad x \in \Omega, \quad t>0, \\
u^{\varepsilon}(x, 0)=u_{0}(x), \quad x \in \Omega ; \quad u^{\varepsilon}(a, t)=u^{\varepsilon}(b, t), \quad u_{x}^{\varepsilon}(a, t)=u_{x}^{\varepsilon}(b, t), \quad t \geq 0,
\end{array}\right.
$$

Choose a mesh size $h:=\Delta x=(b-a) / M$ with $M$ being a positive integer and a time step $\tau:=\Delta t>0$ and denote the grid points and time steps as

$$
x_{j}:=a+j h, \quad j=0,1, \cdots, M ; \quad t_{k}:=k \tau, \quad k=0,1,2, \ldots
$$

Let $u_{j}^{\varepsilon, k}$ be the approximation of $u^{\varepsilon}\left(x_{j}, t_{k}\right)$, and denote $u^{\varepsilon, k}=\left(u_{0}^{\varepsilon, k}, u_{1}^{\varepsilon, k}, \ldots, u_{M}^{\varepsilon, k}\right)^{T} \in \mathbb{C}^{M+1}$ as the numerical solution vectors at $t=t_{k}$. Define the standard finite difference operators

$$
\delta_{t}^{+} u_{j}^{k}=\frac{u_{j}^{k+1}-u_{j}^{k}}{\tau}, \quad \delta_{x}^{+} u_{j}^{k}=\frac{u_{j+1}^{k}-u_{j}^{k}}{h}, \quad \delta_{x}^{2} u_{j}^{k}=\frac{u_{j+1}^{k}-2 u_{j}^{k}+u_{j-1}^{k}}{h^{2}} .
$$

Denote

$$
X_{M}=\left\{v=\left(v_{0}, v_{1}, \ldots, v_{M}\right)^{T} \mid v_{0}=v_{M}, v_{-1}=v_{M-1},\right\} \subseteq \mathbb{C}^{M+1},
$$

equipped with inner products and norms defined as (recall that $u_{0}=u_{M}$ by periodic boundary condition)

$$
\begin{aligned}
& (u, v)=h \sum_{j=0}^{M-1} u_{j} \overline{v_{j}}, \quad\|u\|_{L^{2}}^{2}=(u, u), \quad|u|_{H^{1}}^{2}=\left(\delta_{x}^{+} u, \delta_{x}^{+} u\right), \\
& \|u\|_{H^{1}}=\|u\|_{L^{2}}+|u|_{H^{1}}, \quad\|u\|_{L^{\infty}}=\sup _{0 \leq j \leq M}\left|u_{j}\right| .
\end{aligned}
$$

Then we have for $u, v \in X_{M}$,

$$
\left(-\delta_{x}^{2} u, v\right)=\left(\delta_{x}^{+} u, \delta_{x}^{+} v\right)=\left(u,-\delta_{x}^{2} v\right) .
$$

Following the general CNFD form for the nonlinear Schrödinger equation as in [6, 28], we can get the CNFD discretization as

$$
\left\{\begin{array}{l}
i \delta_{t}^{+} u_{j}^{\varepsilon, k}=-\frac{1}{2} \delta_{x}^{2}\left(u_{j}^{\varepsilon, k}+u_{j}^{\varepsilon, k+1}\right)+G_{\varepsilon}\left(u_{j}^{\varepsilon, k+1}, u_{j}^{\varepsilon, k}\right), \quad j=0, \cdots, M-1, \quad k \geq 0 . \\
u_{j}^{\varepsilon, 0}=u_{0}\left(x_{j}\right), \quad j=0, \cdots, M ; \quad u_{0}^{\varepsilon, k+1}=u_{M}^{\varepsilon, k+1}, \quad u_{-1}^{\varepsilon, k+1}=u_{M-1}^{\varepsilon, k+1},
\end{array}\right.
$$

Here, $G_{\varepsilon}\left(z_{1}, z_{2}\right)$ is defined for $z_{1}, z_{2} \in \mathbb{C}$ as

$$
G_{\varepsilon}\left(z_{1}, z_{2}\right):=\int_{0}^{1} f_{\varepsilon}\left(\theta\left|z_{1}\right|^{2}+(1-\theta)\left|z_{2}\right|^{2}\right) d \theta \cdot \frac{z_{1}+z_{2}}{2}=\frac{F_{\varepsilon}\left(\left|z_{1}\right|^{2}\right)-F_{\varepsilon}\left(\left|z_{2}\right|^{2}\right)}{\left|z_{1}\right|^{2}-\left|z_{2}\right|^{2}} \cdot \frac{z_{1}+z_{2}}{2},
$$


with

$$
\begin{aligned}
& f_{\varepsilon}(\rho)=\lambda \ln (\varepsilon+\sqrt{\rho})^{2} \\
& F_{\varepsilon}(\rho)=\int_{0}^{\rho} f_{\varepsilon}(s) d s=2 \lambda\left(\rho-\varepsilon^{2}\right) \ln (\varepsilon+\sqrt{\rho})-\lambda \rho+2 \varepsilon \lambda \sqrt{\rho} .
\end{aligned}
$$

Then following the analogous arguments of the CNFD method for NLS [6, 28], we can get the conservation properties in the discretized level

$$
\begin{aligned}
& M_{h}\left(u^{\varepsilon, k}\right):=\left\|u^{\varepsilon, k}\right\|_{L^{2}}^{2} \equiv M_{h}\left(u^{\varepsilon, 0}\right), \\
& E_{h}^{\varepsilon}\left(u^{\varepsilon, k}\right):=\left|e^{\varepsilon, k}\right|_{H^{1}}^{2}+h \sum_{j=0}^{M-1} F_{\varepsilon}\left(\left|u_{j}^{\varepsilon, k}\right|^{2}\right) \equiv E_{h}^{\varepsilon}\left(u^{\varepsilon, 0}\right) .
\end{aligned}
$$

\section{Numerical RESUlts}

In this section, we first test the order of accuracy of the regularized Lie-Trotter splitting (LTSP) scheme (2.6), the Strang-splitting (STSP) scheme (2.14) and the CNFD scheme (3.4). Then we apply the Strang-splitting method to investigate some long time dynamics of the LogSE. In practical computation, we impose the periodic boundary condition on $\Omega=(a, b)$ for the RLogSE (1.12).

For the Lie-Trotter and Strang splitting methods, we employ the Fourier pseudo-spectral discretization [8,9] for the spatial variable. Let $M$ be a positive even integer and denote $h=(b-a) / M$ and the grid points $x_{j}=a+j h(0 \leq j \leq M-1)$. Denote by $u^{M, k}$ the discretized solution vector over the grid points $x_{j}(0 \leq j \leq M-1)$ at time $t=t_{k}=k \tau$. Let $\mathcal{F}_{M}$ and $\mathcal{F}_{M}^{-1}$ denote the discrete Fourier transform and its inverse, respectively. With this notation, $\Phi_{A}^{\tau}\left(u^{M, k}\right) 2.3$ can be obtained by

$$
\Phi_{A}^{\tau}\left(u^{M, k}\right)=\mathcal{F}_{M}^{-1}\left(e^{-i \tau\left(\mu^{M}\right)^{2}} \mathcal{F}_{M}\left(u^{M, k}\right)\right),
$$

where

$$
\mu^{M}=\frac{2 \pi}{b-a}\left[0,1, \cdots,\left(\frac{M}{2}-1\right),-\frac{M}{2}, \cdots,-1\right],
$$

and the multiplication of two vectors is taken as point-wise. Moreover, $\Phi_{B}^{\tau}$ can be directly written in physical space.

4.1. Accuracy test. Here, we fix $\lambda=-1$ and $d=1$. We compare the LTSP 2.6), STSP (2.14) and CNFD 3.4 schemes for the following two initial set-ups:

Case I. We consider the smooth Gaussian-type data 1.5 as

$$
u_{0}(x)=\sqrt[4]{-\lambda / \pi} e^{i v x+\frac{\lambda}{2} x^{2}}, \quad x \in \mathbb{R},
$$

where $v$ is a real constant. Indeed, with this initial data and $\Omega=\mathbb{R}, \phi$ and $r$ in $(1.8)-(1.9)$ can be obtained explicitly and the LogSE (1.1) admits a moving Gausson solution (1.6) with velocity $v$ [14, 17.

Case II. We consider the datum in $H^{\vartheta}(\Omega)$ as

$$
u^{M, 0}=\frac{u_{\vartheta}^{M}}{\left\|u_{\vartheta}^{M}\right\|}, \quad u_{\vartheta}^{M}:=\mathcal{F}_{M}^{-1}\left(\left|\mu^{M}\right|^{-\vartheta} \mathcal{F}_{M}\left(\mathcal{U}^{M}\right)\right), \quad\left(\left|\mu^{M}\right|^{-\vartheta}\right)_{l}= \begin{cases}\left|\mu_{l}^{M}\right|^{-\vartheta}, & \text { if } \mu_{l}^{M} \neq 0 \\ 0, & \text { if } \mu_{l}^{M}=0\end{cases}
$$


where

$$
\mathcal{U}^{M}:=\operatorname{rand}(M, 1)+i \operatorname{rand}(M, 1) \in \mathbb{C}^{M},
$$

with $\operatorname{rand}(M, 1)$ returning $M$ uniformly distributed random numbers between 0 and 1 . For typical initial values, see Fig. 4.1.
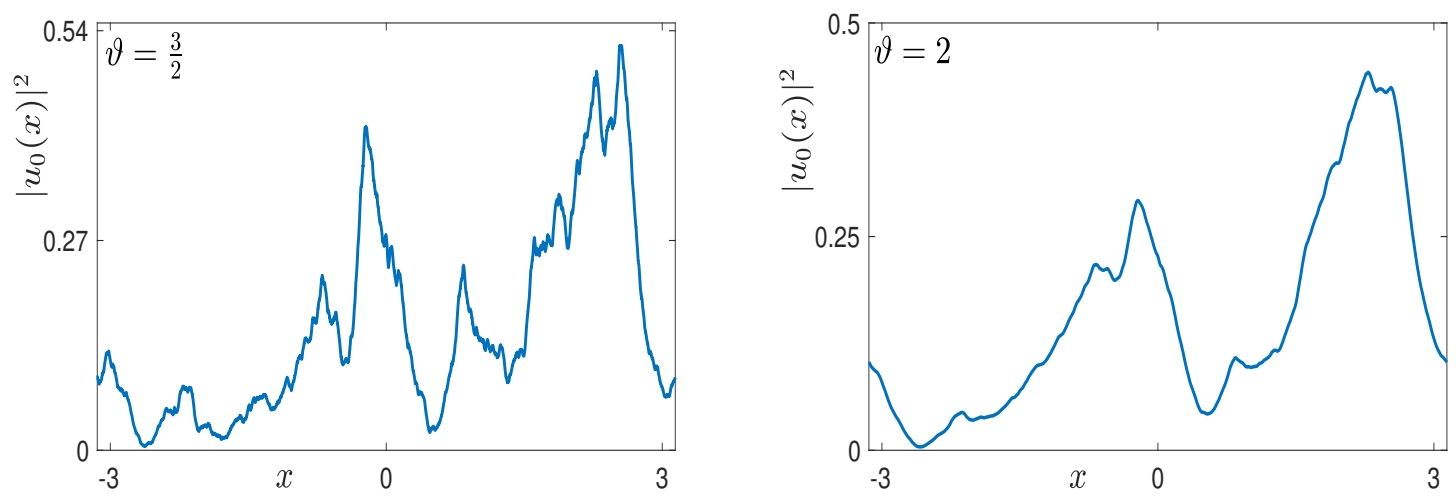

Figure 4.1. Initial data 4.2 for $\vartheta=\frac{3}{2}$ (left) and $\vartheta=2$ (right).

The RLogSE $(1.12)$ is then solved by CNFD, LTSP and STSP on the domain $\Omega=[-16,16]$ and $\Omega=[-\pi, \pi]$ for Case $I$ and Case II, respectively. To quantify the numerical errors, we introduce the error function

$$
e^{\varepsilon}\left(t_{k}\right)=u^{\varepsilon}\left(\cdot, t_{k}\right)-u^{\varepsilon, k},
$$

where $u^{\varepsilon}$ is the exact solution of the RLogSE 1.12 , while $u^{\varepsilon, k}$ is the numerical solution obtained by the CNFD, LTSP or STSP.

Example 1. We consider the initial data Case $I$ (4.1) with $v=1$. The 'exact' solution $u^{\varepsilon}$ in 4.3 is obtained numerically by the STSP with $\tau=\tau_{e}=: 10^{-6}$ and $h=h_{e}=: \frac{1}{2^{8}}$. For LTSP and STSP, we fix $h=h_{e}$ and vary $\tau=\tau_{k}^{j}=: \frac{10^{1-j}}{10+k}$ for $j=1,2,3$ and $k=0, \cdots, 90$. For CNFD, we vary the mesh size and time step simultaneously under ratio $\tau=\frac{2}{5} h=\tau_{j}=: \frac{2^{-j}}{5}$ for $j=0, \cdots, 7$. Fig. 4.2 shows the errors $\left\|e^{\varepsilon}(1)\right\|_{H^{1}}$ vs time step $\tau$ under different $\varepsilon$ for CNFD, LTSP and STSP schemes. It clearly shows that LTSP/STSP is first/second-order convergent in time while CNFD is second-order convergent in both space and time. In addition, for other initial datum smooth enough (not shown here for brevity), all methods show their classical orders of convergence. The same conclusion applies to $\left\|e^{\varepsilon}(1)\right\|_{L^{2}}$ and $\left\|e^{\varepsilon}(1)\right\|_{L^{\infty}}$. Here the norms $\|\cdot\|_{L^{2}},\|\cdot\|_{H^{1}}$ and $\|\cdot\|_{L^{\infty}}$ are defined as (3.2).

Example 2. We consider the initial data Case $I I$ (4.2). The 'exact' solution $u^{\varepsilon}$ in 4.3 . is obtained numerically by the STSP with $\tau=\tau_{e}=: 10^{-6}$ and $h=h_{e}=: \frac{\pi}{2^{15}}$. For all the methods, we fix $h=h_{e}$ and vary $\tau=\tau_{k}^{j}=: \frac{10^{1-j}}{10+k}$ for $j=1,2,3$ and $k=0, \cdots, 90$. The errors $\left\|e^{\varepsilon}(1)\right\|_{L^{2}}$ and $\left\|e^{\varepsilon}(1)\right\|_{H^{1}}$ of the schemes LTSP, STSP and CNFD for the initial value (4.2) with different values of $\vartheta$ are illustrated in Figs. 4.3 and 4.4 , respectively. From these figures we can see that: (i) For smaller values of $\vartheta$, i.e., when the initial data is not smooth enough, all the errors show a zigzag behavior due to happy error cancelation or accumulation 

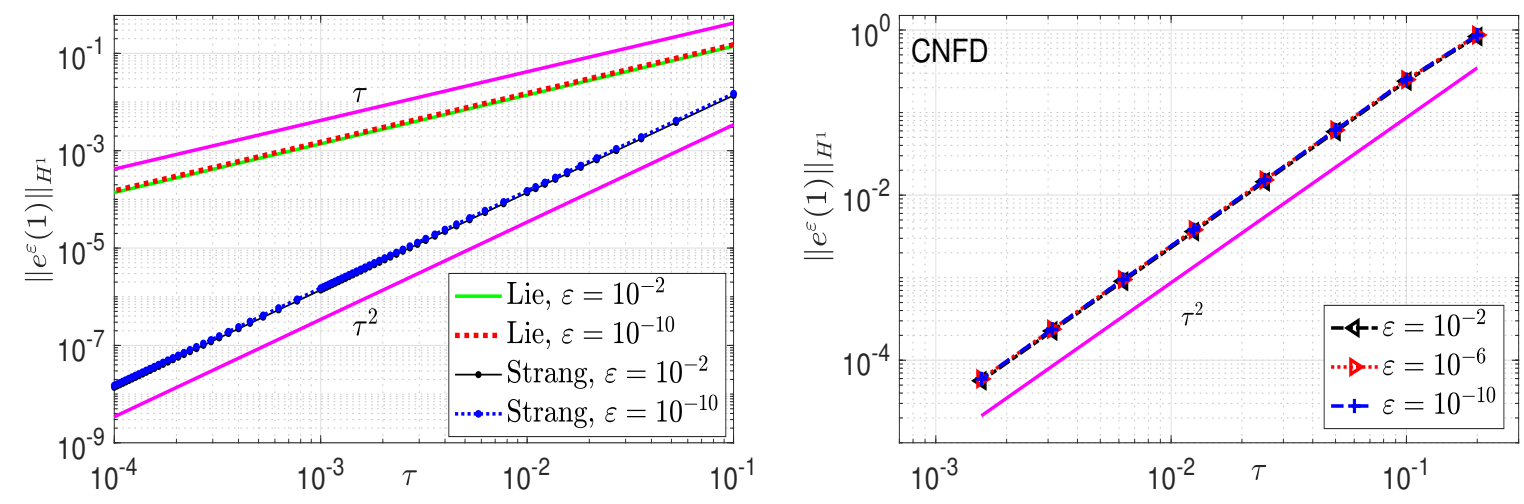

Figure 4.2. Errors $\left\|e^{\varepsilon}(1)\right\|_{H^{1}}$ of LTSP \& STSP (left) and CNFD (right) for Case I.

occurring. Order reduction occurs for all methods in this case. (ii) In $L^{2}$ norm, the LTSP is half-order convergent for $H^{1}$ initial datum (cf. $\vartheta=1$ in Fig. 4.3), which confirms the conclusion in Theorem 2.2. Meanwhile, it is first-order convergent for $\vartheta \geq 2$, which is in line with Remark 2.7. (iii) The STSP is second-order convergent in $L^{2}$ norm for $\vartheta \geq 4$, while the CNFD recovers its second-order convergence only when $\vartheta \geq 5$. (iv) For all the methods, to recover their classical orders of convergence, the initial data is required to be more regular by one additional order when errors are measured in $H^{1}$ norm than in $L^{2}$ norm.

4.2. Applications for long time dynamics. In this section, we apply the STSP method to investigate long time dynamics of LogSE with Gaussian-type initial datum in 1D. To this end, we fix $\varepsilon=10^{-15}, \Omega=[-L, L], h=\frac{1}{16}$ and $\tau=0.001$. The initial data is chosen as

$$
u_{0}(x)=\sum_{k=1}^{N} b_{k} e^{-\frac{a_{k}}{2}\left(x-x_{k}\right)^{2}+i v_{k} x}, \quad x \in \mathbb{R},
$$

where $b_{k}, a_{k}, x_{k}$ and $v_{k}$ are real constants, i.e, the initial data is the sum of $N$ Gaussons 1.5 with velocity $v_{k}$ and initial location $x_{k}$.

Example 3. Here, we let $\lambda=-1, L=1000$. We set $N=2$ in 4.4 and consider the following cases:

(i). $x_{1}=-x_{2}=-5, v_{k}=0, b_{k}=a_{k}=1(k=1,2)$;

(ii). $x_{1}=-x_{2}=-3, v_{k}=0, b_{k}=a_{k}=1(k=1,2)$;

(iii). $v_{1}=v_{2}=2, x_{1}=-x_{2}=-30, b_{k}=a_{k}=1(k=1,2)$;

(iv). $v_{1}=v_{2}=15, x_{1}=-x_{2}=-30, b_{k}=a_{k}=1(k=1,2)$;

(v). $v_{1}=1, v_{2}=0, x_{1}=-40, x_{2}=0, b_{2}=2 b_{1}=1, a_{k}=1(k=1,2)$;

(vi). $v_{1}=4, v_{2}=0, x_{1}=-40, x_{2}=0, b_{2}=2 b_{1}=1, a_{k}=1(k=1,2)$;

(vii). $v_{1}=25, v_{2}=0, x_{1}=-100, x_{2}=0, b_{2}=2 b_{1}=1, a_{k}=1(k=1,2)$;

(viii). $v_{1}=10, v_{2}=0, x_{1}=-50, x_{2}=30, b_{2}=2 b_{1}=1, a_{1}=1.2, a_{2}=0.8$.

Fig. 4.5 shows the evolution of $\sqrt{\left|u^{\varepsilon}(x, t)\right|}, E_{\text {kin }}^{\varepsilon}(t), E_{\text {int }}^{\varepsilon}(t)$ and $E^{\varepsilon}(t)$ as well as the plot of $\left|u^{\varepsilon}(x, t)\right|$ at different time for Cases (i)-(iv). While Fig. 4.6 illustrates those for Cases 

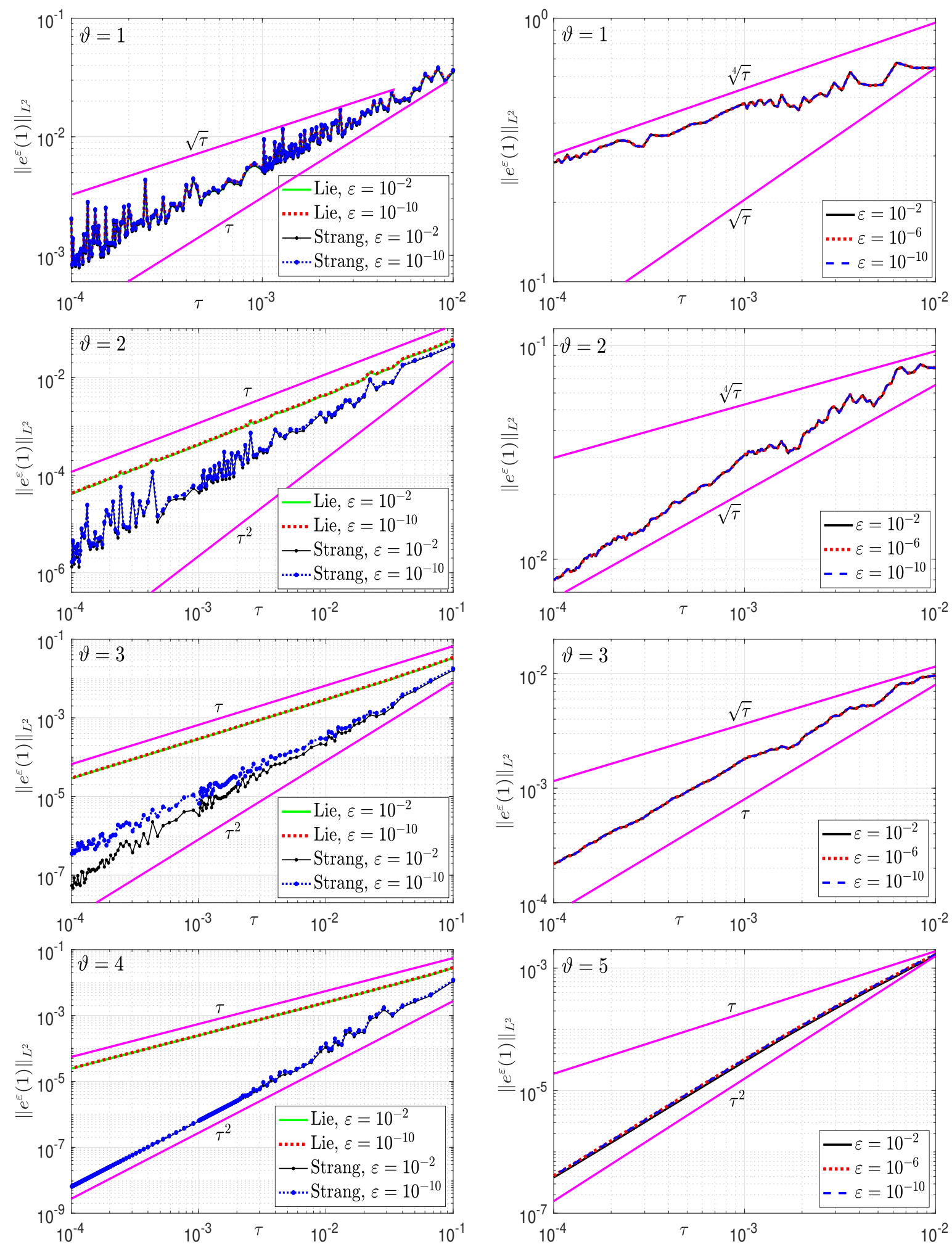

Figure 4.3. Errors $\left\|e^{\varepsilon}(1)\right\|_{L^{2}}$ for the LTSP \& STSP (left) and CNFD (right) in Case $I I$ for different values of $\vartheta$ in 4.2 . 

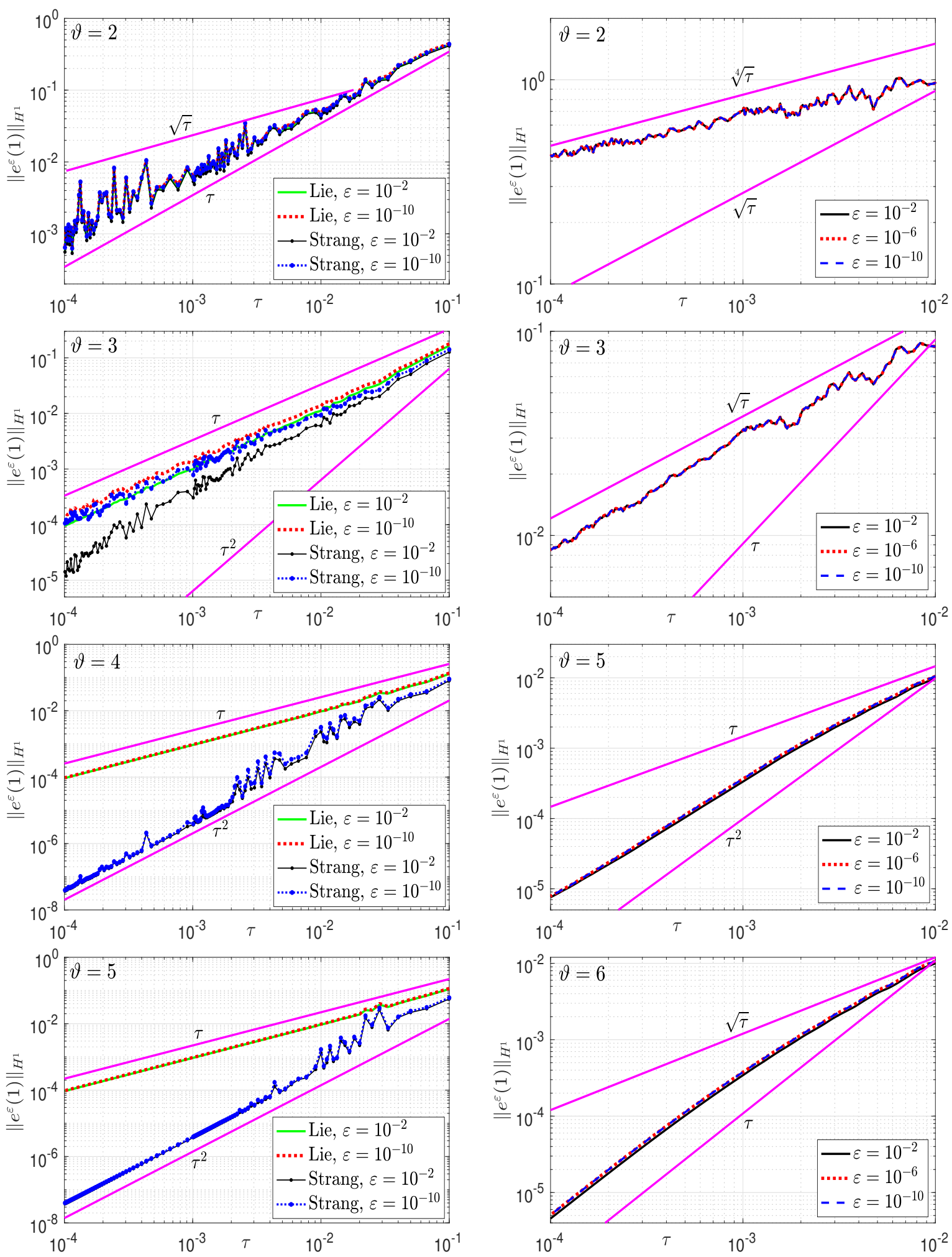

Figure 4.4. Errors $\left\|e^{\varepsilon}(1)\right\|_{H^{1}}$ for the LTSP \& STSP (left) and CNFD (right) in Case $I I$ for different values of $\vartheta$ in (4.2). 
(v)-(viii). Here, the kinetic energy $E_{\text {kin }}^{\varepsilon}$ and interaction energy $E_{\text {int }}^{\varepsilon}$ are defined as:

$$
E_{\text {kin }}^{\varepsilon}(t)=\int_{\Omega}\left|\nabla u^{\varepsilon}(\mathbf{x}, t)\right|^{2} d \mathbf{x}, \quad E_{\text {int }}^{\varepsilon}(t)=E^{\varepsilon}(t)-E_{\text {kin }}^{\varepsilon}(t) .
$$

From these figures we can see that: (1) The total energy is well conserved. (2) For static Gaussons (i.e., $v_{k}=0$ in (4.4)), if they were initially well-separated, the two Gaussons will stay stable as separated static Gaussons (cf. Fig. 4.5 Case i) with density profile unchanged. When they get closer, the two Gaussons contact and undergo attractive interactions. They move to each other, collide and stick together later. Shortly, the two Gaussons separate and swing like pendulum. Small outgoing solitary waves are emitted during the separation of the Gaussons. This wave-emitting 'pendulum motion' becomes faster as time goes on (cf. Fig. 4.5 Case ii). (3) For moving Gaussons, the two Gaussons are transmitted completely through each other and move separately at last. Before they meet, the two Gaussons basically move at constant velocities and preserve their profiles in density exactly if $a_{k}=\lambda$ (While they will move like breathers if $a_{k} \neq \lambda$ (cf. Fig. 4.6 Case viii)). During the interaction, there occurs oscillation. Generally, the larger the relative velocity between the two Gaussons is, the stronger the oscillation is (cf. Fig. 4.5 Cases iii-iv \& Fig. 4.6 Cases vii-viii). After collision, the velocities of Gaussons change (cf. Fig. 4.6 Cases v-vi). The two Gaussons oscillate like breathers and separate completely at last (cf. Fig. 4.5 Cases iii-iv \& Fig. 4.6 Cases vii-viii). In addition, small waves are emitted if the relative velocity is small (cf. Fig. 4.5 Cases ii-iv and Fig. 4.6 Cases v-vi \& viii). (4) For two Gaussons with large relative velocity, their dynamics and interaction are similar to those of the bright solitons in the cubic nonlinear Schrödinger equation [10]. While this is not true for the Gaussons with small relative velocity, in which case additional solitary waves are emitted after collision in the LogSE.

Example 4. Here, we let $\lambda=1$ and $L=10000$. We consider the following three cases of parameters in (4.4):

$$
\begin{aligned}
& \text { (ix). } N=1, v_{1}=10, x_{1}=10, b_{1}=1, a_{1}=1 ; \\
& \text { (x). } N=2, v_{1}=10, v_{2}=0, x_{1}=-100, x_{2}=0, b_{1}=2, b_{2}=1, a_{1}=a_{2}=1 \\
& \text { (xi). } N=2, v_{1}=20, v_{2}=0, x_{1}=-100, x_{2}=0, b_{1}=2, b_{2}=1, a_{1}=a_{2}=1 .
\end{aligned}
$$

Fig. 4.7illustrates the evolution of $\sqrt{\left|u^{\varepsilon}(x, t)\right|}, E_{\text {kin }}^{\varepsilon}, E_{\text {int }}^{\varepsilon}$ and $E^{\varepsilon}$ as well as the plot of $\left|u^{\varepsilon}(x, t)\right|$ at different time for Cases ix-xi. We would conclude from these figures and other numerical experiments not shown here for brevity that: (1) The total energy is conserved well. (2) Unlike the case of $\lambda<0$ where the Gaussons behave like solitary waves, the Gaussians in the case $\lambda>0$ move and spread out (cf. Fig. 4.7). In fact, for a single Gaussian, the analytical solution $u(x, t)$ is given in (1.6). Fig. 4.8 shows the errors of $e(t):=u^{\varepsilon}(\cdot, t)-u(\cdot, t)$ measured in different norms, which again evidence the accuracy of the STSP scheme. In addition, the rate of dispersion of the Gaussians could indeed be estimated for large time dynamics in [17. (3) The dynamics and interaction of two moving Gaussians depend on the relative velocity. They will be separated completely and no solitary waves are emitted if the relative velocity is large enough (cf. Fig. 4.7 Case xi). While if the relative velocity is not large enough, i.e., when they move more slowly than the speed they spread out, the Gaussians will be partially twisted together. Oscillation is created and always there (cf. Fig. 4.7 Case x). This is consistent with the fact that the convergence to a universal Gaussian profile (leaving out the oscillatory aspects, which are not described in general) is very slow, as established in [17 (logarithmic convergence in time). 

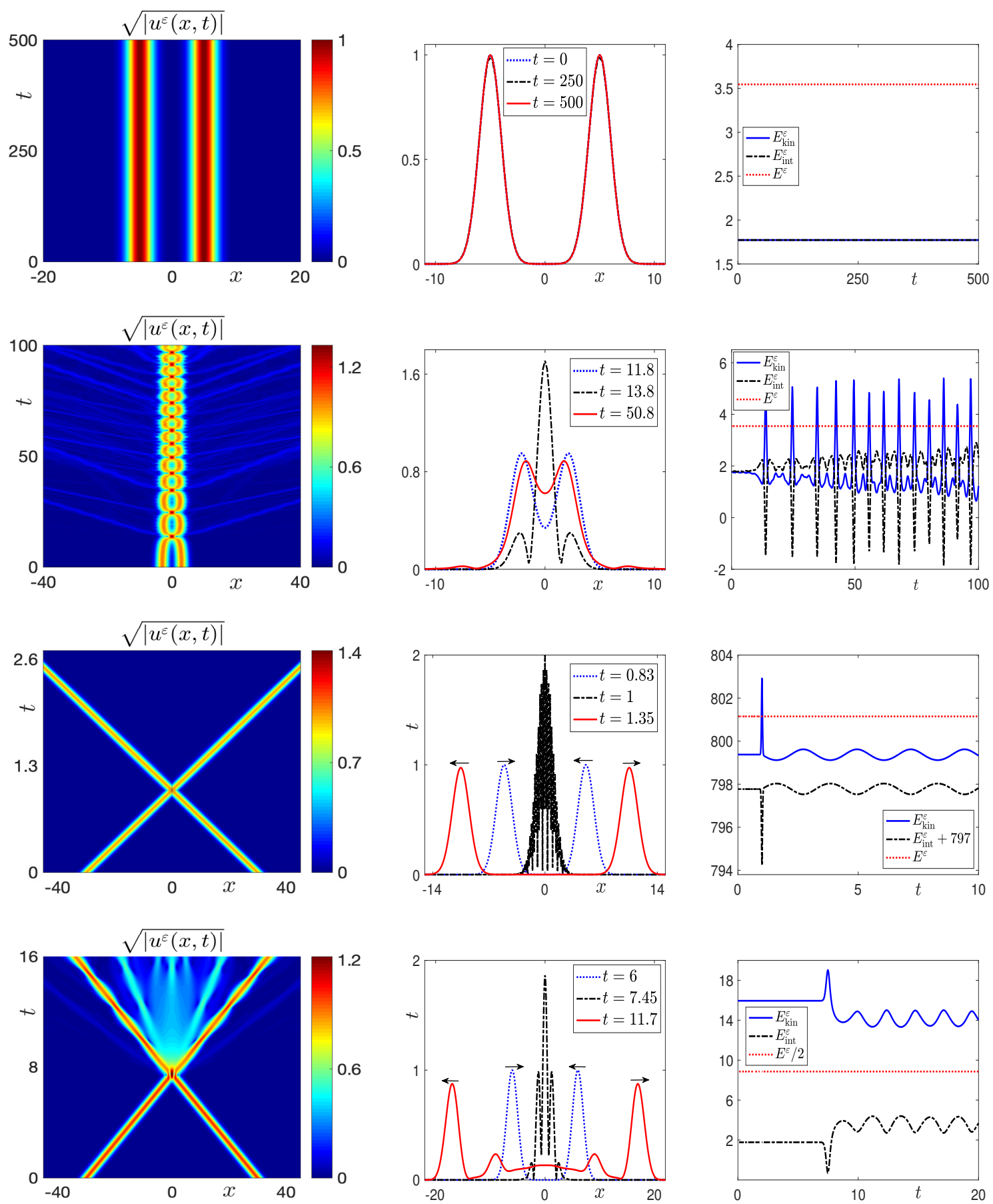

Figure 4.5. Plots of $\sqrt{\left|u^{\varepsilon}(x, t)\right|}$ (first column), $\left|u^{\varepsilon}(x, t)\right|$ at different time (second column) and evolution of the energies (third column) for different parameters in Example 3: Case i-Case iv (from top to bottom). 

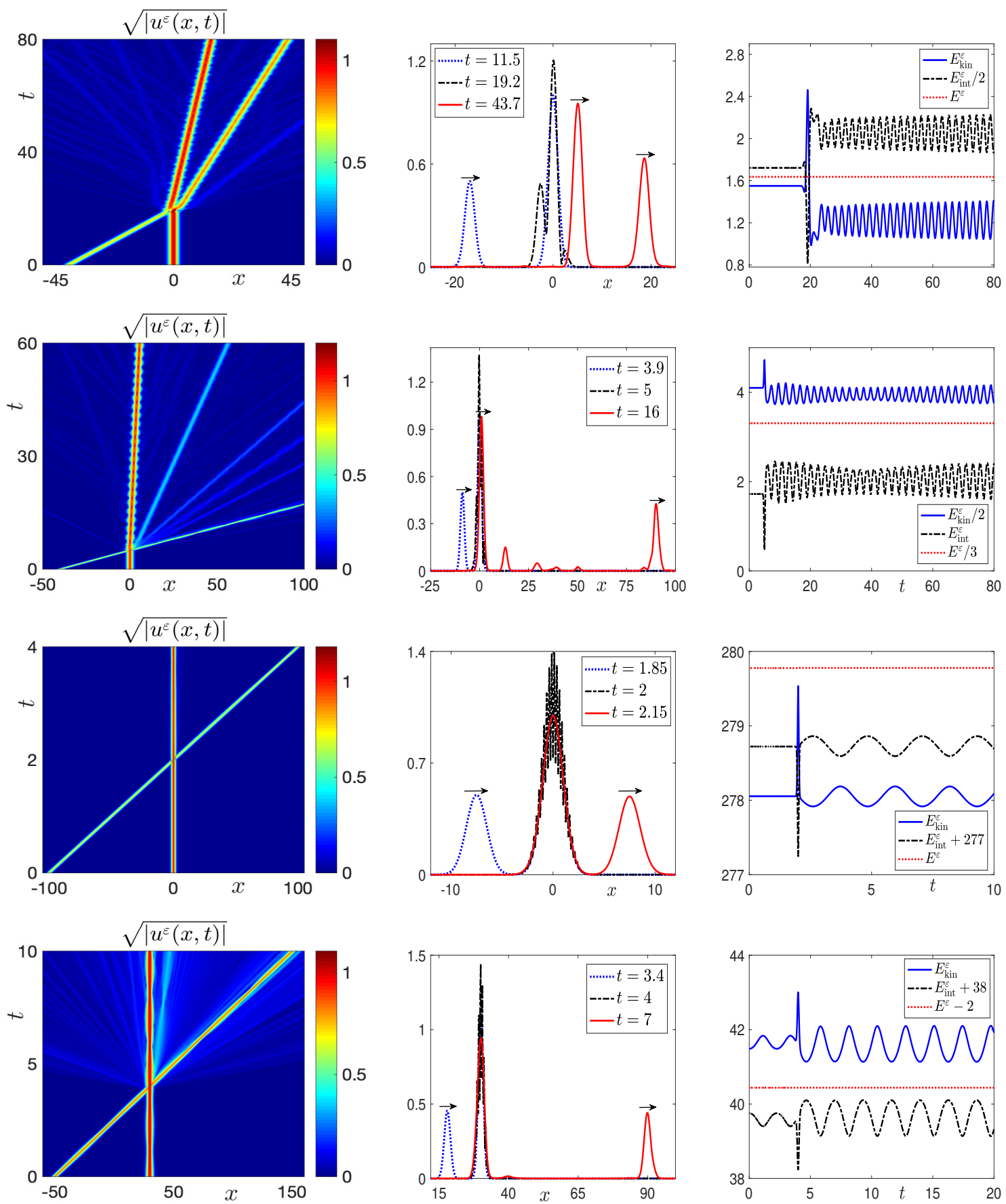

Figure 4.6. Plots of $\sqrt{\left|u^{\varepsilon}(x, t)\right|}$ (first column), $\left|u^{\varepsilon}(x, t)\right|$ at different time (second column) and evolution of the energies (third column) for different parameters in Example 3: Case v-Case viii (from top to bottom). 

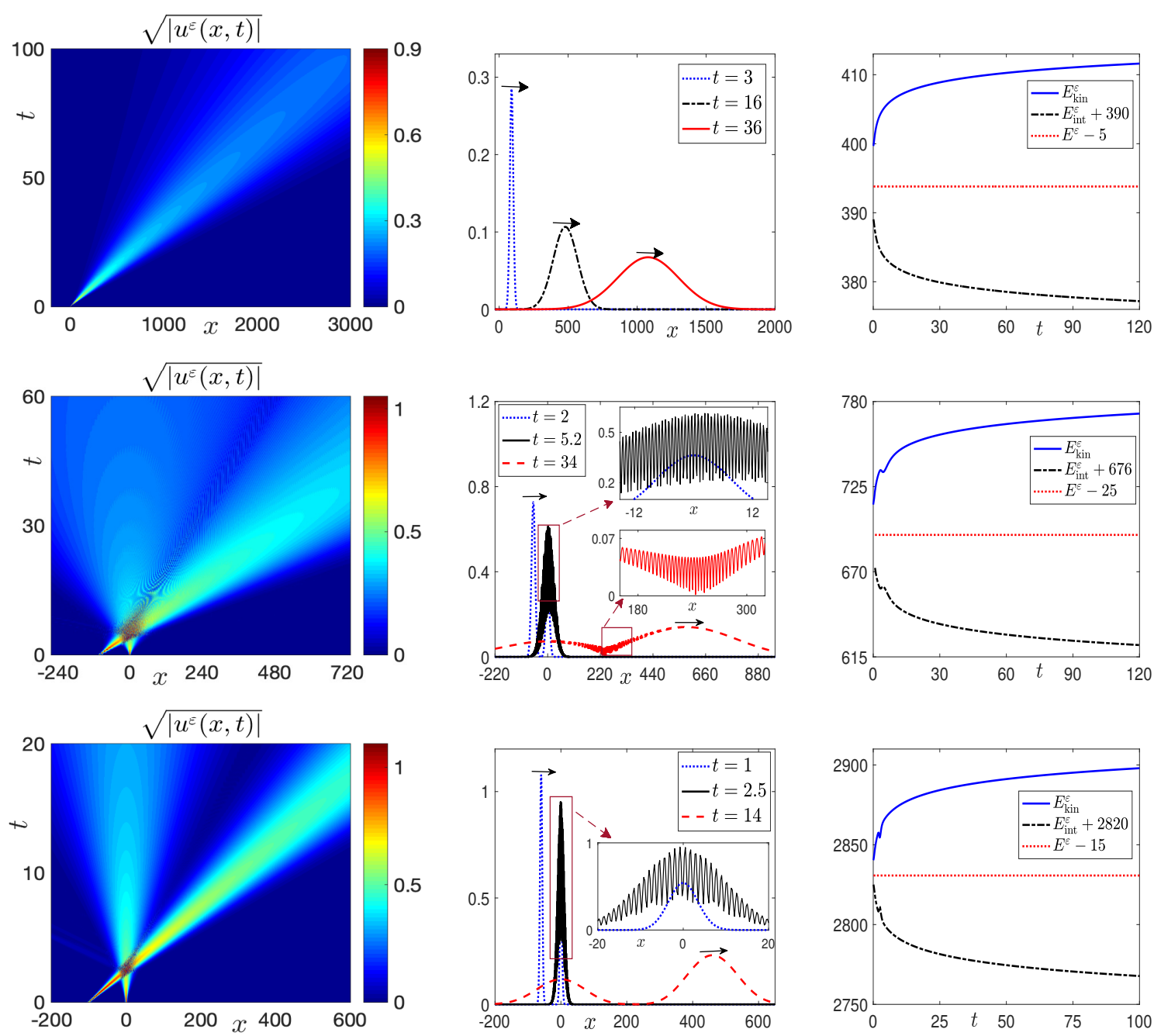

Figure 4.7. Plots of $\sqrt{\left|u^{\varepsilon}(x, t)\right|}$ (first column), $\left|u^{\varepsilon}(x, t)\right|$ at different time (second column) and evolution of the energies (third column) for different parameters in Example 4: Cases ix-xi (from top to bottom).

\section{Conclusion}

We proposed and analyzed the regularized splitting methods and a regularized conservative finite difference method (CNFD) to solve the logarithmic Schrödinger equation (LogSE). For less regular initial setups, reduction of the standard order of accuracy for these methods in temporal direction is proved theoretically for the regularized Lie-Trotter splitting scheme, while also numerically observed for the regularized Strang splitting and CNFD schemes. The method combining the regularized Strang-splitting scheme in time and spectral discretization in space is then applied to investigate the long time dynamics of Gaussians for both positive and negative $\lambda$. It turns out that the interaction of Gaussons in the LogSE is quantitatively 


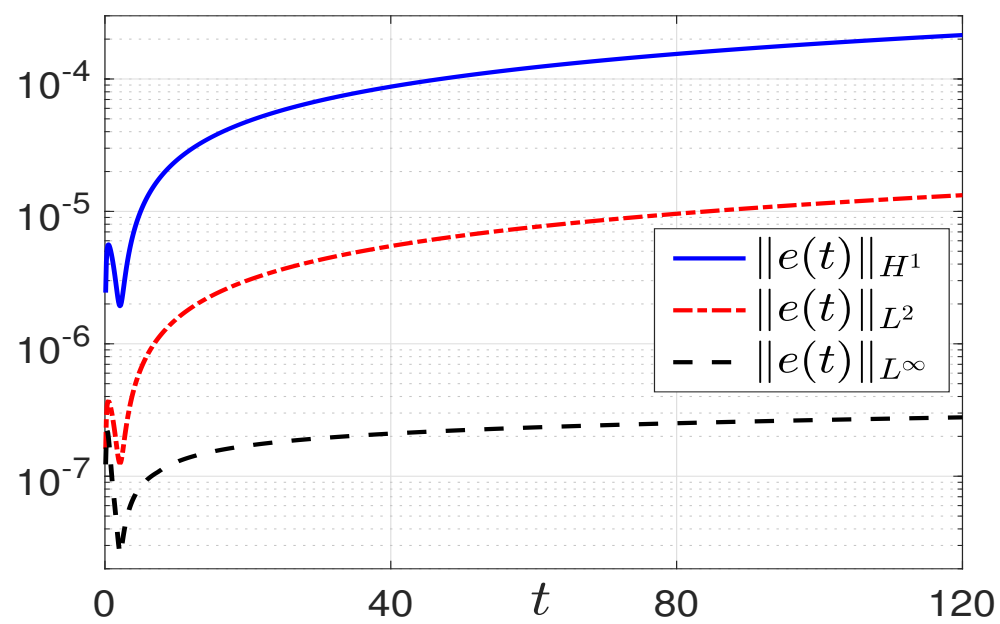

Figure 4.8. Evolution of errors $\left\|e^{\varepsilon}(t)\right\|_{H^{2}},\left\|e^{\varepsilon}(t)\right\|_{L^{2}}$ and $\left\|e^{\varepsilon}(t)\right\|_{L^{\infty}}$ for Case (ix) in Example 4.

similar as the interaction of bright solitons in the cubic nonlinear Schrödinger equation. However, there are also some qualitatively different phenomena such as the breather-like dynamics and the spreading-out behavior when $\lambda<0$ and when $\lambda>0$ in the LogSE, respectively. Our numerical results demonstrate rich and complicated dynamical phenomena in the LogSE.

\section{REFERENCES}

[1] G. D. Akrivis, Finite difference discretization of the cubic Schrödinger equation, IMA J. Numer. Anal., 13 (1993), pp. 115-124.

[2] G. D. Akrivis, V. A. Dougalis, and O. A. Karakashian, On fully discrete Galerkin methods of second-order temporal accuracy for the nonlinear Schrödinger equation, Numer. Math., 59 (1991), pp. 3153.

[3] X. Antoine, W. Bao, And C. Besse, Computational methods for dynamics of the nonlinear Schrödinger/Gross-Pitaevskii equations, Comput. Phys. Commun., 184 (2013), pp. 2621-2633.

[4] A. H. Ardila, Orbital stability of Gausson solutions to logarithmic Schrödinger equations, Electron. J. Differ. Eq., 335 (2016), pp. 1-9.

[5] A. V. Avdeenkov and K. G. Zloshchastiev, Quantum Bose liquids with logarithmic nonlinearity: Selfsustainability and emergence of spatial extent, J. Phys. B: Atomic, Molecular Optical Phys., 44 (2011), p. 195303.

[6] W. BAO AND Y. CAI, Optimal error estimates of finite difference methods for the Gross-Pitaevskii equation with angular momentum rotation, Math. Comp., 82 (2013), pp. 99-128.

[7] W. BaO, R. CARles, C. Su, AND Q. TANG, Error estimates of a regularized finite difference method for the logarithmic Schrödinger equation, arXiv: 1803.10068.

[8] W. BaO, D. Jaksch, AND P. A. MARKowich, Numerical solution of the Gross-Pitaevskii equation for Bose-Einstein condensation, J. Comput. Phys., 187 (2003), pp. 318-342.

[9] W. BAo, S. Jin, AND P. A. MARKowich, On time-splitting spectral approximations for the Schrödinger equation in the semiclassical regime, J. Comput. Phys., 175 (2002), pp. 487-524.

[10] W. BAO, Q. TANG, AND Z. XU, Numerical methods and comparison for computing dark and bright solitons in the nonlinear Schrödinger equation, J. Comput. Phys., 235 (2013), pp. 423-445.

[11] C. BEsse, A relaxation scheme for the nonlinear Schrödinger equation, SIAM J. Numer. Anal., 42 (2004), pp. 934-952. 
[12] C. Besse, B. Bidégaray, And S. Descombes, Order estimates in time of splitting methods for the nonlinear Schrödinger equation, SIAM J. Numer. Anal., 40 (2002), pp. 26-40.

[13] I. BiaŁynicki-Birula And J. Mycielski, Nonlinear wave mechanics, Ann. Physics, 100 (1976), pp. 6293.

[14] - Gaussons: Solitons of the logarithmic Schrödinger equation, Special issue on solitons in physics, Phys. Scripta, 20 (1979), pp. 539-544.

[15] H. Buljan, A. Šiber, M. Soljačić, T. Schwartz, M. Segev, and D. Christodoulides, Incoherent white light solitons in logarithmically saturable noninstantaneous nonlinear media, Phys. Rev. E, 68 (2003), p. 036607.

[16] R. CARLES, On Fourier time-splitting methods for nonlinear Schrödinger equations in the semiclassical limit, SIAM J. Numer. Anal., 51 (2013), pp. 3232-3258.

[17] R. Carles and I. Gallagher, Universal dynamics for the defocusing logarithmic Schrödinger equation, Duke Math. J., 167 (2018), pp. 1761-1801.

[18] R. Carles And C. Gallo, On Fourier time-splitting methods for nonlinear Schrödinger equations in the semi-classical limit II. Analytic regularity, Numer. Math., 136 (2017), pp. 315-342.

[19] T. Cazenave, Stable solutions of the logarithmic Schrödinger equation, Nonlinear Anal., 7 (1983), pp. $1127-1140$.

[20] — Semilinear Schrödinger equations, vol. 10 of Courant Lecture Notes in Mathematics, New York University Courant Institute of Mathematical Sciences, New York, 2003.

[21] T. Cazenave and A. Haraux, Équations d'évolution avec non linéarité logarithmique, Ann. Fac. Sci. Toulouse Math. (5), 2 (1980), pp. 21-51.

[22] T. Cazenave And P.-L. Lions, Orbital stability of standing waves for some nonlinear Schrödinger equations, Commun. Math. Phys., 85 (1982), pp. 549-561.

[23] Q. Chang, E. JiA, AND W. Sun, Difference schemes for solving the generalized nonlinear Schrödinger equation, J. Comput. Phys., 148 (1999), pp. 397-415.

[24] A. Debussche And E. FAou, Modified energy for split-step methods applied to the linear Schrödinger equation, SIAM J. Numer. Anal., 47 (2009), pp. 3705-3719.

[25] S. Descombes And M. Thalhammer, The Lie-Trotter splitting for nonlinear evolutionary problems with critical parameters: a compact local error representation and application to nonlinear Schrödinger equations in the semiclassical regime, IMA J. Numer. Anal., 33 (2012), pp. 722-745.

[26] E. FAOU AND B. GRÉBERT, Hamiltonian interpolation of splitting approximations for nonlinear PDEs, Found. Comput. Math., 11 (2011), pp. 381-415.

[27] L. GAUCKLER AND C. LuBICH, Splitting integrators for nonlinear Schrödinger equations over long times, Found. Comput. Math., 10 (2010), pp. 275-302.

[28] R. Glassey, Convergence of an energy-preserving scheme for the Zakharov equations in one space dimension, Math. Comp., 58 (1992), pp. 83-102.

[29] P. Guerrero, J. L. López, And J. Nieto, Global $H^{1}$ solvability of the $3 D$ logarithmic Schrödinger equation, Nonlinear Anal. Real World Appl., 11 (2010), pp. 79-87.

[30] B. Guo, The convergence of numerical method for nonlinear Schrödinger equation, J. Comput. Math., 4 (1986), pp. 121-130.

[31] T. Hansson, D. Anderson, and M. Lisak, Propagation of partially coherent solitons in saturable logarithmic media: A comparative analysis, Phys. Rev. A, 80 (2009), p. 033819.

[32] M. HAYASHI, A note on the nonlinear Schrödinger equation in a general domain, Nonlinear Analysis, 173 (2018), pp. 99-122.

[33] E. F. Hefter, Application of the nonlinear Schrödinger equation with a logarithmic inhomogeneous term to nuclear physics, Phys. Rev. A, 32 (1985), pp. 1201-1204.

[34] P. Henning And D. Peterseim, Crank-Nicolson Galerkin approximations to nonlinear Schrödinger equations with rough potentials, Math. Models Methods Applied Sci., 27 (2017), pp. 2147-2184.

[35] E. S. Hernandez And B. Remaud, General properties of Gausson-conserving descriptions of quantal damped motion, Physica A, 105 (1980), pp. 130-146.

[36] L. I. IGNAT, A splitting method for the nonlinear Schrödinger equation, J. Diff. Eq., 250 (2011), pp. 30223046.

[37] O. Karakashian and C. Makridakis, A space-time finite element method for the nonlinear Schrödinger equation: the continuous Galerkin method, SIAM J. Numer. Anal., 36 (1999), pp. 1779-1807. 
[38] W. Krolikowski, D. Edmundson, And O. BAng, Unified model for partially coherent solitons in logarithmically nonlinear media, Phys. Rev. E, 61 (2000), pp. 3122-3126.

[39] C. LubICH, On splitting methods for Schrödinger-Poisson and cubic nonlinear Schrödinger equations, Math. Comp., 77 (2008), pp. 2141-2153.

[40] P. A. Markowich, P. Pietra, AND C. Pohl, Numerical approximation of quadratic observables of Schrödinger-type equations in the semi-classical limit, Numer. Math., 81 (1999), pp. 595-630.

[41] S. D. Martino, M. Falanga, C. Godano, and G. Lauro, Logarithmic Schrödinger-like equation as a model for magma transport, Europhys. Lett., 63 (2003), pp. 472-475.

[42] R. I. McLachlan and G. R. W. Quispel, Splitting methods, Acta Numerica, 11 (2002), pp. 341-434.

[43] M. Robinson, G. Fairweather, And B. Herbst, On the numerical solution of the cubic Schrödinger equation in one space variable, J. Comput. Phys., 104 (1993), pp. 277-284.

[44] M. Squassina AND A. Szulkin, Multiple solutions to logarithmic Schrödinger equations with periodic potential, Calc. Var. Partial Differ. Equ., 54 (2015), pp. 585-597.

[45] T. R. TAha And M. I. Ablowitz, Analytical and numerical aspects of certain nonlinear evolution equations. II. Numerical, nonlinear Schrödinger equation, J. Comput. Phys., 55 (1984), pp. 203-230.

[46] K. TANAKA And C. Zhang, Multi-bump solutions for logarithmic Schrödinger equations, Calc. Var. Partial Differ. Eq., 56 (2017), p. 33.

[47] M. Thalhammer, M. Caliari, and C. Neuhauser, High-order time-splitting Hermite and Fourier spectral methods, J. Comput. Phys., 228 (2009), pp. 822-832.

[48] K. Yasue, Quantum mechanics of nonconservative systems, Annals Phys., 114 (1978), pp. 479-496.

[49] Y. ZHU, Implicit difference schemes for the generalized non-linear Schrödinger system, J. Comput. Math., 1 (1983), pp. 116-129.

[50] K. G. Zloshchastiev, Logarithmic nonlinearity in theories of quantum gravity: Origin of time and observational consequences, Grav. Cosmol., 16 (2010), pp. 288-297.

(W. Bao) Department of Mathematics, National University of Singapore, Singapore 119076

E-mail address: matbaowz@nus.edu.sg

$U R L:$ http://www.math.nus.edu.sg/ bao/

(R. Carles) Univ Rennes, CNRS, IRMAR - UMR 6625, F-35000 Rennes, France

E-mail address: Remi.Carles@math.cnrs.fr

$U R L:$ http://carles.perso.math.cnrs.fr/

(C. Su) Department of Mathematics, University of Innsbruck, Innsbruck 6020, Austria

E-mail address: sucm13@163.com

(Q. Tang) School of Mathematics, Sichuan University, Chengdu 610064, People's Republic of CHINA

E-mail address: qinglin_tang@scu.edu.cn

URL: http://math.scu.edu.cn/info/1013/3088.htm 